Pure and Applied Mathematics Quarterly

Volume 3, Number 1

(Special Issue: In honor of

Robert MacPherson, Part 3 of 3 )

$61-105,2007$

\title{
Towards Non-Reductive Geometric Invariant Theory
}

Brent Doran and Frances Kirwan

To Bob MacPherson on his 60th birthday

\begin{abstract}
We study linear actions of algebraic groups on smooth projective varieties $X$. A guiding goal for us is to understand the cohomology of "quotients" under such actions, by generalizing (from reductive to non-reductive group actions) existing methods involving Mumford's geometric invariant theory (GIT). We concentrate on actions of unipotent groups $\mathrm{H}$, and define sets of stable points $X^{s}$ and semistable points $X^{s s}$, often explicitly computable via the methods of reductive GIT, which reduce to the standard definitions due to Mumford in the case of reductive actions. We compare these with definitions in the literature. Results include (1) a geometric criterion determining whether or not a ring of invariants is finitely generated, (2) the existence of a geometric quotient of $X^{s}$, and (3) the existence of a canonical "enveloping quotient" variety of $X^{s s}$, denoted $X / / H$, which (4) has a projective completion given by a reductive GIT quotient and (5) is itself projective and isomorphic to $\operatorname{Proj}\left(k[X]^{H}\right)$ when $k[X]^{H}$ is finitely generated.
\end{abstract}

\section{Contents}

1. Introduction 62

2. Motivation 65

2.1. Moduli spaces $\quad 65$

2.2. Affine geometry 67

2.3. Classical invariant theory 68

3. GIT Basics 68

3.1. Comparison of reductive and non-reductive 69

Received January 26, 2006. 
3.2. Geometric and categorical quotients 71

3.2.1. GIT for reductive group actions on projective varieties 72

3.2.2. Reductive GIT and quasi-projective varieties 73

4. Generalizing GIT: Intrinsic viewpoints 75

4.1. Global approaches 76

4.1.1. Naive stability 76

$\begin{array}{ll}\text { 4.1.2. Quotients by free actions } & 77\end{array}$

4.2. Gluing local quotients $X_{f} / / H \quad 79$

5. Generalizing GIT: Induced reductive actions - from $H$ to $G \quad 82$

5.1. Stability for $G \times{ }_{H} X$

5.2. Completions and reductive envelopes 85

5.3. Main Theorem and Corollaries $\quad 89$

5.3.1. Constructing strong reductive envelopes $\quad 89$

5.3.2. Important case: $H$-action extends to $G$-action 89

5.3.3. Consequences for finite generation 91

6. Example: $n$ unordered points on $\mathbb{P}^{1} \quad 98$

6.1. $n=3$ and $4 \quad 99$

$\begin{array}{ll}\text { 6.2. Cohomology of quotients } & 101\end{array}$

References 103

\section{INTRODUCTION}

Geometric invariant theory (GIT) is a powerful theoretical and computational tool for the study of reductive algebraic group actions. On the theory side, it provides a good notion of a quotient of an affine or projective variety; many key moduli spaces admit a description as a GIT quotient. More computationally, information about the geometry of projective GIT quotients, in particular about their cohomology (indeed, algebraic K-theory, motivic cohomology or any oriented cohomology theory $[2,35,14,6,13])$, can often be extracted using not much more than the theory of weights of linear representations. As an added bonus, the "stable" structures identified by GIT tend to have very nice (differential) geometric properties [57]. A drawback is that GIT requires the group which acts to be reductive, whereas many interesting problems in moduli theory, affine 
geometry, and classical invariant theory are rooted in non-reductive actions. This paper addresses the question of how to develop an effective version of GIT for general affine algebraic group actions, including some of the difficulties, context, and motivating problems, with the eventual goal of computing the cohomology of quotients of nonsingular projective varieties by such actions.

Two key properties of actions of a reductive group $G$ on an affine variety $X$ are crucial to GIT. Firstly, by a theorem of Nagata, the ring of invariants $k[X]^{G}$ is finitely generated. Secondly, given any two disjoint closed $G$-invariant subvarieties of $X$, there exists an invariant function which separates them. These lead to the existence of a "good categorical quotient" $\pi: X \rightarrow X / / G=\operatorname{Spec}\left(k[X]^{G}\right)$, along with a distinguished open subset $X^{s}$ of stable orbits such that $\pi\left(X^{s}\right) \subseteq$ $X / / G$ is a "geometric quotient", that is, an orbit space with nice properties. Quotients of more general varieties $X$ (equipped with linearizations of the group actions) are constructed by patching together suitable open affine pieces to get a categorical quotient $X / / G$ of the semistable subset $X^{s s}$ of $X$, with an open subset $X^{s} / G \subseteq X / / G$ giving a geometric quotient of $X^{s}$.

Less essential, but still very useful, is a third property of reductive actions, that every invariant extends to an invariant of the ambient affine (or projective) space. This effectively reduces GIT to the study of representations of reductive groups on the affine (or projective) space itself. Fourthly and finally, the quotients $X / / G$ of affine or projective varieties are again affine or projective, respectively, with the quotient maps $X^{s s} \rightarrow X / / G$ surjective.

Each of these properties fails for non-reductive groups. Most famously, examples of non-finitely generated rings of invariants (counter-examples to Hilbert's Fourteenth Problem) were first discovered by Nagata [49]. One might hope, however, that a good generalization of GIT to non-reductive group actions would, as in the reductive theory, satisfy the following properties:

- It would depend only on the data of a $G$-linearization of $X$; that is, a $G$ equivariant embedding of $X$ into an affine or projective space equipped with a linear action of $G$, together with a lift of the action of $G$ to the (homogeneous) coordinate ring $k[X]$.

- It would use the invariants in $k[X]$ to separate as many closed orbits as possible.

- It would provide notions of stable and semistable subsets $X^{s}$ and $X^{s s}$ with a canonical $G$-invariant morphism $X^{s s} \rightarrow X / / G$ (ideally a categorical quotient) restricting to a geometric quotient of $X^{s}$.

- It would have a good 'change of groups' formalism relating $X / / H$ and $\left(G \times_{H} X\right) / / G$ if $H$ is a closed subgroup of $G$.

With this is mind, we discuss some generalizations of GIT to non-reductive group actions, including various notions of "stable" and "semistable" points. A 
direct approach is to patch together "nice enough" affine opens which admit finitely generated rings of invariants. Another method is to transfer the problem to reductive GIT by considering the associated reductive $G$-action on (projective completions of) $G \times_{H} X$; given a linearized $H$-action on $X$ in $\mathbb{P}^{n}$, we can consider

$$
G \times_{H} X \subseteq G \times_{H} \mathbb{P}^{n} \cong(G / H) \times \mathbb{P}^{n},
$$

for $G$ a reductive group extending the action of $H$ on $\mathbb{P}^{n}$, and reductive GIT on $G \times{ }_{H} \mathbb{P}^{n}$ or an appropriate projective completion $\overline{G \times{ }_{H} \mathbb{P}^{n}}$. In each approach, and conceptually perhaps this is the crucial point, only finitely many invariants are really being used to define the quotients. Somewhat remarkably, when properly formulated these approaches are compatible, and most of the conditions above are satisfied - though the "enveloping quotient" $X^{s s} \rightarrow X / / H$ which we obtain fails to be a categorical quotient in general, and its image may not be a subvariety of $X / / H$ but only a dense constructible subset. As a byproduct we obtain a geometric criterion for deciding whether or not the ring of invariants $k[X]^{H}$ is finitely generated. When $X$ is projective and $k[X]^{H}$ is finitely generated then $X / / H=\operatorname{Proj}\left(k[X]^{H}\right)$ is a projective variety; more generally we obtain projective completions $\overline{X / / H}$ of $X / / H$ which are themselves reductive GIT quotients $\overline{G \times{ }_{H} X} / / G$ and hence, in principle, amenable to standard methods for understanding their geometry and topology.

Each affine algebraic group $H$ has a unipotent radical $H_{u}$, which by definition is non-trivial precisely when $H$ is non-reductive. Effectively, one may first quotient by the action of $H_{u}$, and then by the induced action of the reductive group $H / H_{u}$, provided that the unipotent quotient is sufficiently canonical to inherit an induced linear action of the reductive group. So what really must be understood is unipotent actions, and we shall concentrate on these.

For convenience, although this is hardly necessary, we work over an algebraically closed base field $k$ of characteristic 0 , which in examples will be taken to be $\mathbb{C}$. By "variety" we mean an integral scheme of finite type over $k$. Except where otherwise stated, we assume $X$ is a projective variety.

This paper is intended to be sufficiently self-contained that readers with minimal background knowledge will find it accessible, so it is at times, by necessity or design, somewhat informal. After this introduction $\S 2$ discusses some problems in mathematics where non-reductive actions appear: moduli spaces, exotic affine spaces, Hilbert's fourteenth problem, and so forth. $\S 3$ is a brief summary of the idea behind geometric invariant theory - using invariants to parametrize orbits - followed by an explanation of how key facts about reductive actions fail for non-reductive ones, and a summary of the main definitions and results of reductive GIT needed later. $\S 4$ discusses various 'intrinsic' ways to describe open subsets on which non-reductive actions admit nice quotients, and compares these to existing definitions and approaches in the literature. $\$ 5$ develops the 
approach to non-reductive GIT via reductive GIT on an auxiliary space; there are three main results. Theorem 5.3.1 summarizes the relationships between the different notions of stable points, semistable points, and their "quotients", while Theorem 5.3.5 provides a stronger conclusion when a particular criterion is satisfied, which can often be arranged in practice (for example, when $k[X]^{H}$ is finitely generated). With these in mind, Definition 5.3.7 sets our notion of stable and semistable points. Theorem 5.3.18 gives a geometric criterion for deciding when a ring of invariants is finitely generated, relating it to a (non-effective) stability condition in reductive GIT. Finally $\S 6$ discusses a family of $\mathbb{C}^{+}$-actions as a straightforward example and computes the (intersection) cohomology of their enveloping quotients $X / / H$.

The authors thank Bob MacPherson for his support and inspiration, as well as Aravind Asok and Charles Doran for helpful conversations and comments. Indeed, it was in the first author's time as Bob's student that this circle of ideas first began to take shape.

\section{Motivation}

Reductive group actions are of great significance in algebraic geometry, but non-reductive actions appear in many important problems. Over $\mathbb{C}$ a group $G$ is reductive if and only if it is the complexification of a maximal compact subgroup $K$, and many nice properties can obtained by exploiting this underlying compactness. The simplest example here is of course the complexification $\mathbb{C}^{*}$ of the circle $S^{1}$, and more generally $G L(n ; \mathbb{C})$ viewed as the complexification of the unitary group $U(n)$; in contrast $\mathbb{C}^{+}$has no nontrivial compact subgroups. Given the ubiquity of translation actions, it is not surprising that non-reductive groups appear in so many problems, though sometimes they are well hidden. Two very recent examples include Bridgeland-Douglas stability conditions in derived categories of sheaves interpreted, in a special motivating case, as stability for matrix factorizations using non-reductive actions [29, 58], and the study of hyperbolic varieties via Griffiths-Green jet bundles, after Demailly et al. [54]; some more classical examples are given below.

2.1. Moduli spaces. In the preface to the first edition of [48], Mumford states that his goal is "to construct moduli schemes for various types of algebraic objects" and that this problem "appears to be, in essence, a special and highly non-trivial case" of the problem of constructing orbit spaces for algebraic group actions. More precisely, when a family of objects with parameter space $S$ has the local universal property for a given moduli problem, and a group acts on $S$ such that objects parametrized by points in $S$ are equivalent if and only if the points lie in the same orbit, then the construction of a coarse moduli space is equivalent to the construction of a categorical quotient which is an orbit space for the action 
(cf. [50, Proposition 2.13]). There are many cases of moduli problems involving non-reductive group actions. Here are a few examples.

- Moduli of singularities (or modules over the local ring of a singularity) as studied in $[23,24]$; here the rough idea is that translation actions arise from unfoldings of singularities.

- Moduli of suitable maps; for example, degree $d$ maps between projective spaces or with additional constraints (see [16, pp. 80-83]).

- Moduli of hypersurfaces in toric varieties.

This last example generalizes the very classical moduli problem of hypersurfaces in projective space $\mathbb{P}^{n}$. There the space of hypersurfaces of degree $d$ is parametrized by $\mathbb{P}\left(S y m^{d}\left(\mathbb{C}^{n+1}\right)\right)$ and the equivalence is given by the natural linear action of the reductive group $S L(n+1 ; \mathbb{C})$. There is a canonical (categorical) projective GIT quotient with an open subset which is a coarse moduli space for stable hypersurfaces. For a general toric variety $X$, the analogous parameter space is also a projective space, but the action is a linear action of a non-reductive group. Such spaces arise, for example, in the study of moduli spaces of Calabi-Yau varieties and mirror symmetry [7]. Lacking a theory of non-reductive quotients, the standard trick is to study an associated "simplified" moduli space which arises as a quotient of a torus action; it is a branched cover of the actual moduli space and understanding the geometry of its compactification is complicated and not entirely naturally related to the actual moduli problem.

Example 2.1.1. Let $X$ be the weighted projective plane $\mathbb{P}(1,1,2)$, with homogeneous coordinates $x, y, z$, and consider the moduli problem of weighted degree 4 hypersurfaces (which are genus 1 curves) in this toric variety $X$. The relevant group action is that of the automorphism group of $X$, which lifts to a semidirect product of the unipotent group $\left(\mathbb{C}^{+}\right)^{3}$ acting via

$$
[x: y: z] \mapsto\left[x: y: z+\lambda x^{2}+\mu x y+\nu y^{2}\right] \quad \text { for }(\lambda, \mu, \nu) \in\left(\mathbb{C}^{+}\right)^{3}
$$

and the reductive group $G L(2 ; \mathbb{C}) \times G L(1 ; \mathbb{C})$ acting on the $(x, y)$ coordinates and the $z$ coordinate. A basis for weighted degree 4 polynomials is

$$
\left\{x^{4}, x^{3} y, x^{2} y^{2}, x y^{3}, y^{4}, x^{2} z, x y z, y^{2} z, z^{2}\right\}
$$


With respect to this basis, the $\left(\mathbb{C}^{+}\right)^{3}$ action is linearly represented as:

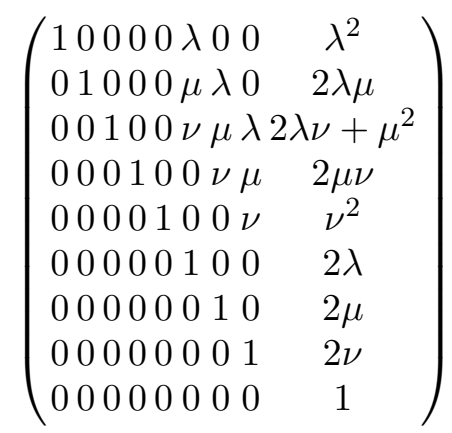

2.2. Affine geometry. An abundant supply of translation symmetries means that non-reductive actions occur everywhere in affine geometry. In particular the automorphism group $\operatorname{Aut}\left(\mathbb{A}^{n}\right)$ of $n$-dimensional affine space is a surprisingly rich and mysterious structure, which ties into many of the famous questions in the area. To name one example, the Jacobian Conjecture is equivalent to the statement that an automorphism $F=\left(F_{1}, \ldots, F_{n}\right)$ of $\mathbb{A}^{n}$ naturally induces a set of $n$ locally nilpotent derivations $\frac{d}{d F_{1}}, \ldots, \frac{d}{d F_{n}}$, i.e., a unipotent action on $\mathbb{A}^{n}[15, \S 2.2]$. Another instance is the existence of exotic affine spaces (varieties diffeomorphic but not algebraically isomorphic to $\mathbb{A}^{n}$ ), where constructions of examples directly or implicitly make heavy use of unipotent actions. Perhaps the cleanest to write down is the Russell cubic three-fold:

$$
X=\left\{(w, x, y, z) \in \mathbb{C}^{4} \mid x^{2} w+x+y^{2}+z^{3}=0\right\},
$$

which Makar-Limanov [44] proves is exotic effectively by showing it has a smaller set of $\mathbb{C}^{+}$actions than $\mathbb{C}^{3}$ does (more precisely, the intersection of all the invariant subrings for the $\mathbb{C}^{+}$actions is not simply the constants). Perhaps more strikingly, arbitrary dimensional families of non-isomorphic exotic affine spaces can be constructed using quotients by free unipotent actions [59, 1].

Example 2.2.1. Let $\phi_{t}: \mathbb{C}^{5} \rightarrow \mathbb{C}^{5}$ be the quadratic free $\mathbb{C}^{+}$action given by:

$$
\phi_{t}\left(w_{1}, w_{2}, w_{3}, w_{4}, w_{5}\right)=\left(w_{1}, w_{2}, w_{3}+t w_{1}, w_{4}+t w_{2}, w_{5}+t\left(1+w_{1} w_{4}-w_{2} w_{3}\right)\right) .
$$

This action on $\mathbb{C}^{5}$ has a geometric quotient which is diffeomorphic to $\mathbb{C}^{4}$ and quasi-affine but not affine. The quotient can be thought of as the complement to the cotangent space at a point in the cotangent bundle to $S^{4}$, where the bundle is embedded as an affine quadric hypersurface.

Moreover Winkelmann [60] has shown that the study of the coordinate rings of quasi-affine varieties over any field $k$ (which are, of course, not necessarily finitely generated as $k$-algebras) is precisely the study of the invariant subrings of affine varieties under affine algebraic group actions (indeed $k^{+}$actions). 
Theorem 2.2.2. (Winkelmann) If $R$ is an integrally closed $k$-algebra then the following are equivalent:

- there exists a quasi-affine irreducible, reduced $k$-variety $X$ such that $R \cong$ $k[X]$;

- there exists an irreducible, reduced $k$-variety $X$ and a subgroup $G$ of Aut $(X)$ such that $R \cong k[X]^{G}$;

- there exists an affine irreducible, reduced $k$-variety $X$ and a regular action of $G=k^{+}$on $X$ such that $R \cong k[X]^{G}$.

2.3. Classical invariant theory. The fourteenth of Hilbert's problems posed at the 1900 International Congress of Mathematicians was the following question: If an algebraic group acts linearly on a polynomial ring in finitely many variables, is the ring of invariants always finitely generated? The answer is yes for reductive groups (and for some non-reductive groups, in particular for $\mathbb{C}^{+}$), but Nagata [49] showed that the answer is no, in general, though counterexamples have not been easy to find. Nagata's original counterexample was an action of $\left(\mathbb{C}^{+}\right)^{13}$. Much later Mukai found an action of $\left(\mathbb{C}^{+}\right)^{3}$ where the ring of invariants is not finitely generated [45], and made further generalizations in [46, 47]; see [8] for some very recent related results on $\left(\mathbb{C}^{+}\right)^{2}$-actions. Popov [51] used Nagata's counterexample to show that if an algebraic group $G$ is not reductive then there is an affine $G$-variety $X$ such that $k[X]^{G}$ is not finitely generated.

\section{GIT BASICS}

If $X$ is a normal quasi-projective $G$-variety, then there exists a $G$-linearization [11][Theorem 7.3] for the $G$-action on $X$; that is, a $G$-equivariant embedding in some projective space together with a lift of the $G$-action to the (homogeneous) coordinate ring ${ }^{1}$. The main goal of geometric invariant theory is to provide a natural algebraic variety (depending only on the choice of linearization) which parametrizes $G$-orbits in an affine or projective variety $X$ by using invariant functions (or sections) in the (homogeneous) coordinate ring $A=k[X]$. When the ring $A^{G}$ of all invariants is a finitely generated $k$-algebra, as is the case if $G$ is reductive, we obtain an affine variety $\operatorname{Spec}\left(A^{G}\right)$ and a morphism

$$
F: X=\operatorname{Spec}(A) \longrightarrow \operatorname{Spec}\left(A^{G}\right)
$$

for affine $X$, or

$$
F: X=\operatorname{Proj}(A) \rightarrow \operatorname{Proj}\left(A^{G}\right)
$$

for projective $X$, induced by the inclusion $A^{G} \rightarrow A$. In the affine case $F$ is a dominant morphism, whereas in the projective case it is just a dominant rational map; $F$ is not defined precisely where all the invariants vanish, known as the

\footnotetext{
${ }^{1}$ Note that if $X$ is not normal then this is not necessarily true; for example, the nodal cubic curve is a $\mathbb{C}^{*}$-equivariant completion of $\mathbb{C}^{*}$, but not of any linear $\mathbb{C}^{*}$ orbit.
} 
unstable set. If $X$ is affine and $\left\{f_{i}\right\}_{1 \leq i \leq n}$ is a finite generating set for $A^{G}$, then one may realize $F$ as the morphism to affine space $\left(f_{1}, \ldots, f_{n}\right): X \rightarrow \mathbb{A}^{n}$; more precisely, this morphism is the composition of $\mathrm{F}$ with an embedding of $\operatorname{Spec}\left(A^{G}\right)$ in $\mathbb{A}^{n}$. A similar statement holds in the projective case, except the grading on the image must be compatible with the degrees of the $f_{i}$ so that the image lies in a weighted projective space.

When $A^{G}$ is not finitely generated we can still consider $\operatorname{Spec}\left(A^{G}\right)$ and $\operatorname{Proj}\left(A^{G}\right)$ but only as schemes, not varieties.

Definition 3.0.1. Let $X$ be a quasi-projective variety, and $L$ an ample line bundle endowed with a lifting of the $G$-action. A finite separating set of invariants is a collection of invariant sections $\left\{f_{1}, \ldots, f_{n}\right\}$ of positive tensor powers of $L$ such that, if $x, y$ are any two points of $X$, and $f$ is an arbitrary invariant section of $L^{\otimes k}$ for some $k>0$, then

$$
(f(x)=f(y), \forall f) \Leftrightarrow\left(f_{i}(x)=f_{i}(y), \forall i=1, \ldots, n\right) .
$$

Remark 3.0.2. Other authors consider a similar notion. See [9, Section 2.3.2] for a comparison.

Since $X$ is Noetherian one sees that finite separating sets of invariants always exist (the analogous result in [9] is Theorem 2.3.15). When $X$ is affine any finite collection of invariant functions $f_{1}, \ldots, f_{n}$ defines a $G$-invariant map $\left(f_{1}, \ldots, f_{n}\right): X \rightarrow \mathbb{A}^{n}$ (likewise in the projective case), and one could hope that when $\left\{f_{1}, \ldots, f_{n}\right\}$ is a finite separating set of invariants the image of $\left(f_{1}, \ldots, f_{n}\right)$ might be a variety independent of the choice of $\left\{f_{1}, \ldots, f_{n}\right\}$. Unfortunately this is not true in general, but a refinement of the idea does work (cf. Proposition 4.2.9 below).

For a general affine $G$ over a characteristic zero field, there is a semi-direct product decomposition $G \cong G_{r} \ltimes G_{u}$ into a reductive subgroup $G_{r}$ and the unipotent radical $G_{u}$ of $G$, and the corresponding rings of invariants satisfy $A^{G}=\left(A^{G_{u}}\right)^{G_{r}}$. (Note that the characteristic zero hypothesis and ensuing semi-direct product structure are just conveniences; in any characteristic $G_{u}$ is a normal unipotent subgroup of $G$ such that $G / G_{u}$ is reductive, and then $\left.A^{G}=\left(A^{G_{u}}\right)^{G / G_{u}}\right)$. So the key to non-reductive geometric invariant theory lies in unipotent actions.

3.1. Comparison of reductive and non-reductive. To see how non-reductive actions differ from reductive ones, it suffices to work with unipotent groups. Unipotent actions have several appealing features.

- Every orbit for a unipotent group action on a quasi-affine variety is closed [53], so unipotent actions are always closed in the sense of Mumford; this is helpful for GIT since invariants cannot distinguish an orbit from another orbit in its closure. 
- Every homogeneous space of a unipotent group, and hence every orbit of a unipotent group action, is isomorphic to an affine space [33, Proposition 8.4.1].

- A connected unipotent group over a characteristic 0 field has no proper finite subgroups, hence a unipotent action has no points with non-trivial but finite isotropy groups. (This follows from embedding the unipotent group as a subgroup of upper triangular matrices in some linear representation.)

However there are also several ways in which unipotent actions behave less well than reductive actions. We list some key properties of reductive invariant theory which make GIT so effective, and follow them with unipotent counter-examples.

Proposition 3.1.1. [48, Corollary 1.2] Let $X$ be an affine variety with a reductive $G$-action. Given two disjoint $G$-invariant closed subvarieties $Y_{1}$ and $Y_{2}$ in $X$, there exists an invariant $f \in k[X]^{G}$ such that $f\left(Y_{1}\right)=0$ and $f\left(Y_{2}\right)=1$.

Proposition 3.1.2. [48, Theorem 1.1(3)] Let $X$ be a G-invariant affine subvariety of $\mathbb{A}^{n}$ where $G$ is reductive and acts on $\mathbb{A}^{n}$. Then given $f \in k[X]^{G}$ there exists an extension $F$ of $f$ to an invariant on $\mathbb{A}^{n}$. More precisely, if $I_{X}$ is the defining ideal of $X$, then $k[X]^{G}=k\left[\mathbb{A}^{n}\right]^{G} / I_{X} \cap k\left[\mathbb{A}^{n}\right]^{G}$.

Example 3.1.3. Let $\mathbb{C}^{+}$act on $X=\operatorname{Sym}^{2}(V)$ via its inclusion as upper triangular matrices in the defining two-dimensional representation $V$ of $S L(2 ; \mathbb{C})$.

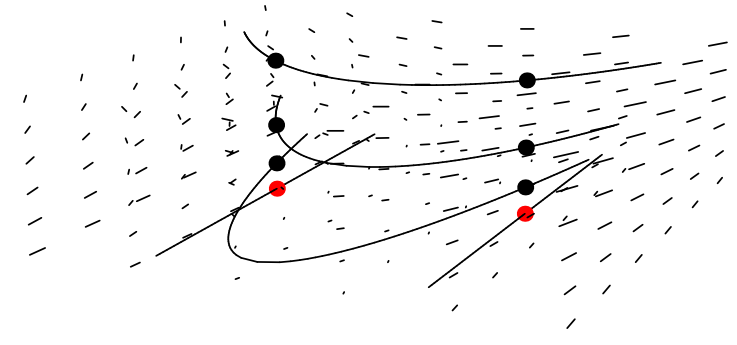

FiguRE $1 . \mathbb{C}^{+}$-orbits in $X=\operatorname{Sym}^{2}(V)$

Here the invariants are easy to describe with respect to the usual coordinates $x_{0}, x_{1}, x_{2}$ on $X=\operatorname{Sym}^{2}(V)$ : we have $A^{\mathbb{C}^{+}}=\mathbb{C}\left[x_{0}, x_{1}^{2}-x_{0} x_{2}\right]$. Observe that, even though this is an action on an affine space, the invariants do not separate all closed orbits - the pairs of lines $\left\{x_{0}=0, x_{1}= \pm a\right\}$ for any given $a \neq 0$ are not distinguished, nor are any of the fixed points of the line $\left\{x_{0}=0, x_{1}=0\right\}$. Furthermore, observe that not all invariants of the hyperplane defined by $x_{0}=0$ lift to invariants of $X \cong \mathbb{C}^{3}$; for example $x_{1}$ is an invariant function of the $\mathbb{C}^{+}$ 
action on the hyperplane, but does not extend to an invariant of the action on $X$.

This example illustrates as well two important principles: firstly, that nonclosed orbits are not the only source of non-separated orbit spaces; and secondly, that set-theoretic freeness does not imply scheme-theoretic freeness, as the action of $\mathbb{C}^{+}$on the complement of the fixed point line $\left\{x_{0}=0, x_{1}=0\right\}$ is not even separated - because the parabolic orbits in Figure 1 degenerate into pairs of disjoint orbits in the $\left(x_{0}, x_{1}\right)$-plane - let alone scheme-theoretically free [ 48 , Definition 0.8] (that is, set-theoretically free and proper [13, §6.3 Lemma 8]).

Proposition 3.1.4. [11, Theorem 3.3] (Nagata) Let $G$ be a reductive group acting on an affine variety $X=\operatorname{Spec}(A)$. Then $A^{G}$ is a finitely generated $k$-algebra.

Example 3.1.5 (Nagata counter-example). Consider the $n$-fold direct sum $V^{\oplus n}$ of the defining representation $V$ for $S L(2 ; \mathbb{C})$, and look at the subgroup of $G L\left(V^{\oplus n}\right)$ corresponding to the direct sum of the $n$ Borel subgroups (each one is a semidirect product of $\mathbb{C}^{+}$and $\left.\mathbb{C}^{*}\right)$ :

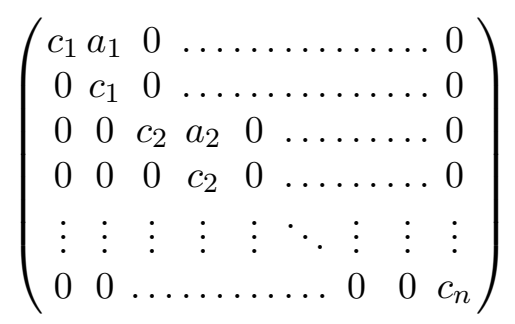

with $a_{i} \in \mathbb{C}$ and $c_{i} \in \mathbb{C}^{*}$. Demand that $\prod_{i} c_{i}=1$. Then for appropriate $n$ and a closed subgroup $G$ cut out by an appropriate set of linear conditions on the $a_{i}$, the ring of invariants $\mathbb{C}\left[x_{1}, \ldots, x_{n}, y_{1}, \ldots, y_{n}\right]^{G}$ is not finitely generated [11, Theorem 4.3].

Proposition 3.1.6. Let $G$ be a reductive group acting on an affine variety $X=$ $\operatorname{Spec}(A)$, or respectively a projective variety $X=\operatorname{Proj}(A)$. Then the quotient map $q: \operatorname{Spec}(A) \longrightarrow \operatorname{Spec}\left(A^{G}\right)$, or respectively $q: \operatorname{Proj}(A)^{s s} \longrightarrow \operatorname{Proj}\left(A^{G}\right)$, is surjective.

Example 3.1.7. This proposition says that the GIT quotient of an affine (projective) variety by a reductive group action is affine (projective), whereas in contrast Example 2.2.1 provides an affine variety whose quotient by a non-reductive group action is quasi-affine but not affine.

3.2. Geometric and categorical quotients. Recall (from [50, Chapter 2, §4], for example) that when a group $G$ acts on a variety $X$ a categorical quotient of $X$ by $G$ is a morphism $\phi: X \rightarrow Y$ from $X$ to a variety $Y$ which is $G$-invariant (that is, constant on $G$-orbits) and has the property that any other $G$-invariant morphism $\tilde{\phi}: X \rightarrow \tilde{Y}$ factors as $\tilde{\phi}=\chi \circ \phi$ for a unique morphism $\chi: Y \rightarrow \tilde{Y}$. An 
orbit space for the action is a categorical quotient $\phi: X \rightarrow Y$ such that $\phi^{-1}(y)$ is a single $G$-orbit for each $y \in Y$, and a geometric quotient is an orbit space $\phi: X \rightarrow Y$ with the following good properties: it is an affine morphism such that

(i) if $U$ is open in $Y$ then

$$
\phi^{*}: k[U] \rightarrow k\left[\phi^{-1}(U)\right]
$$

induces an isomorphism of $k[U]$ onto $k\left[\phi^{-1}(U)\right]^{G}$, and

(ii) if $W_{1}$ and $W_{2}$ are disjoint closed $G$-invariant subvarieties of $X$ then their images $\phi\left(W_{1}\right)$ and $\phi\left(W_{2}\right)$ in $Y$ are disjoint closed subvarieties of $Y$.

Thus an orbit space is a geometric quotient if and only if it is a good categorical quotient.

3.2.1. GIT for reductive group actions on projective varieties. Let $X$ be a projective variety over $k$ and let $G$ be a reductive group acting on $X$. For Mumford's geometric invariant theory we also require a linearization of the action; that is, a line bundle $L$ on $X$ and a $\operatorname{lift}^{2}$ of the action of $G$ to $L$. When $L$ is ample we can assume without essential loss of generality that for some projective embedding $X \subseteq \mathbb{P}^{n}$ the action of $G$ on $X$ extends to an action on $\mathbb{P}^{n}$ given by a representation

$$
\rho: G \rightarrow G L(n+1 ; k),
$$

where $L$ is the hyperplane line bundle on $\mathbb{P}^{n}$. There is an induced action of $G$ on the homogeneous coordinate ring

$$
A=k[X] \stackrel{\text { def }}{=} \bigoplus_{k \geq 0} H^{0}\left(X, L^{\otimes k}\right)
$$

of $X$, which, when $X \subseteq \mathbb{P}^{n}$ as above, is the quotient $k\left[x_{0}, \ldots, x_{n}\right] / \mathcal{I}_{X}$ of the polynomial ring $k\left[x_{0}, \ldots, x_{n}\right]$ by the ideal $\mathcal{I}_{X}$ generated by the homogeneous polynomials vanishing on $X$. The subring $A^{G}$ of $A$ consisting of the elements of $A$ left invariant by $G$ is a graded $k$-algebra, which by Nagata's theorem is finitely generated because $G$ is reductive [49], so we can define $X / / G$ (or $X / /{ }_{L} G$ when the dependence on the linearization $L$ is to be made explicit) to be the variety $\operatorname{Proj}\left(A^{G}\right)$ associated to this ring of invariants $A^{G}$. The inclusion of $A^{G}$ in $A$ defines a rational map $\phi$ from $X$ to $X / / G$, but because there may be points of $X \subseteq \mathbb{P}_{n}$ where every nonconstant $G$-invariant homogeneous polynomial vanishes, this map will not in general be a morphism.

We define the set $X^{s s}(L)$ (abbreviated to $X^{s s}$ when there is no risk of confusion) of semistable points for the action of $G$ on $X$ with respect to the ample linearization $L$ to consist of those $x \in X$ for which there exists some $k>0$ and $s \in H^{0}\left(X, L^{\otimes k}\right)^{G}$ not vanishing at $x$. Then the rational map $\phi$ restricts to a

\footnotetext{
${ }^{2}$ When there is no risk of confusion we will use $L$ to denote the linearization as well as the underlying line bundle.
} 
surjective $G$-invariant morphism from the open subset $X^{s s}$ of $X$ to the projective variety $X / / G$, and $\phi: X^{s s} \rightarrow X / / G$ is a categorical quotient for the action of $G$ on $X^{s s}$. Set-theoretically, $X / / G$ is the quotient of $X^{s s}$ by the equivalence relation for which $x$ and $y$ in $X^{s s}$ are equivalent if and only if the closures $\overline{O_{G}(x)}$ and $\overline{O_{G}(y)}$ of the $G$-orbits of $x$ and $y$ meet in $X^{s s}$.

In order to obtain a geometric quotient, we define a stable point for the linear action of $G$ on $X$ to be a point $x$ of $X^{s s}$ with a neighborhood in $X^{s s}$ such that every $G$-orbit meeting this neighborhood is closed in $X^{s s}$, and is of maximal dimension equal to the dimension of $G$ (a "properly stable point" in the sense of [48, Definition 1.8]). If $U$ is any $G$-invariant open subset of the set $X^{s}=X^{s}(L)$ of stable points of $X$, then $\phi(U)$ is an open subset of $X / / G$ and the restriction $\left.\phi\right|_{U}: U \rightarrow \phi(U)$ of $\phi$ to $U$ is a universal geometric quotient (it remains a geometric quotient under base change) for the action of $G$ on $U$. In particular, there is a geometric quotient $X^{s} / G=\phi\left(X^{s}\right)$ for the action of $G$ on $X^{s}$, and if $X^{s}$ is nonempty then $X / / G$ can be thought of as a projective completion of the quasiprojective variety $X^{s} / G$ :

$$
\begin{aligned}
& \begin{array}{ccccc}
X^{s} & \underset{1}{\subseteq} & X^{s s} & \stackrel{\subseteq}{\subseteq} & X \\
& \text { open } & & \text { open } & \\
\downarrow & & \downarrow & &
\end{array} \\
& X^{s} / G \subseteq X^{s s} / \sim=X / / G \text {. } \\
& \text { open }
\end{aligned}
$$

Remark 3.2.1. Each of $X^{s}, X^{s s}$, and $X / / G$ remains unchanged if the linearization $L$ is replaced by $L^{\otimes m}$ for any $m>0$.

Remark 3.2.2. When $X$ is a connected nonsingular projective variety and $G$ is a connected reductive group acting on $X$ with generic stabilizer having dimension 0 , then a $G$-invariant open subset $U$ of $X$ is proper and there exists a quasiprojective geometric quotient $\phi: U \rightarrow Y$ if and only if $U \subseteq X^{s}(L)$ for some linearization $L$ of the action of $G$ (see [48, Converse 1.13]).

Remark 3.2.3. Let $T$ be a maximal torus of a reductive group $G$ acting linearly on $X$. Then the subsets $X^{s s}$ and $X^{s}$ of $X$ are characterised by the following properties (essentially the Hilbert-Mumford criteria for stability and semistability) which make them easy to identify:

(i) A point $x \in X$ is semistable (respectively stable) for the linear action of $G$ on $X$ if and only if $g x$ is semistable (respectively stable) for the action of $T$ on $X$ for every $g \in G$.

(ii) If the maximal torus $T$ of $G$ acts diagonally on $X \subseteq \mathbb{P}_{n}$ with weights $\alpha_{0}, \ldots, \alpha_{n}$, then a point $x=\left[x_{0}, \ldots, x_{n}\right] \in X$ is semistable (respectively stable) for 
the action of $T$ if and only if the convex hull

$$
\operatorname{Conv}\left\{\alpha_{i}: x_{i} \neq 0\right\}
$$

in $\mathbf{t}^{*}$ contains 0 (respectively contains 0 in its interior) where $\mathbf{t}^{*}$ is the vector space dual of the Lie algebra of $T$.

Example 3.2.4. Consider the linear action of $\mathbb{C}^{*}$ on $X=\mathbb{P}^{n}$, with respect to the hyperplane line bundle on $\mathbb{P}^{n}$, where the linearization $L_{0}$ is given by the representation

$$
t \mapsto \operatorname{diag}\left(t^{2}, t^{2}, \ldots, t^{2}, 1\right)
$$

of $\mathbb{C}^{*}$ in $G L(n+1 ; \mathbb{C})$. The same action of $\mathbb{C}^{*}$ on $\mathbb{P}^{n}$ has other linearizations with respect to the hyperplane line bundle; let $L_{+}$denote the linearization given by the representation

$$
t \mapsto \operatorname{diag}\left(t^{3}, t^{3}, \ldots, t^{3}, t\right)
$$

of $\mathbb{C}^{*}$ in $G L(n+1 ; \mathbb{C})$ and let $L_{-}$denote the linearization given by the representation

$$
t \mapsto \operatorname{diag}\left(t, t, \ldots, t, t^{-1}\right)
$$

of $\mathbb{C}^{*}$ in $G L(n+1 ; \mathbb{C})$. Then

$$
X^{s s}\left(L_{+}\right)=X^{s}\left(L_{+}\right)=\emptyset,
$$

while

and

$$
X^{s s}\left(L_{0}\right)=\mathbb{C}^{n}, X^{s}\left(L_{0}\right)=\emptyset,
$$

$$
X^{s s}\left(L_{-}\right)=X^{s}\left(L_{-}\right)=\mathbb{C}^{n} \backslash\{0\} .
$$

3.2.2. Reductive GIT and quasi-projective varieties. If a $G$-action on a variety $X$ has a categorical quotient $\phi: X \rightarrow Y$ then its restriction to a $G$-invariant open subset of $X$ is not necessarily a categorical quotient for the action of $G$ on $U$, as the following simple example shows.

Example 3.2.5. Let the multiplicative group $\mathbb{C}^{*}$ of $\mathbb{C}$ act on $\mathbb{C}^{n}$ as multiplication by scalars. Since the origin lies in the closure of every orbit, it follows that every $G$-invariant morphism $\phi: \mathbb{C}^{n} \rightarrow Y$ is constant and hence that the constant map from $\mathbb{C}^{n}$ to a point is a categorical quotient for the action. However the restriction of this constant map to $\mathbb{C}^{n} \backslash\{0\}$ is not a categorical quotient, since the natural map $\mathbb{C}^{n} \backslash\{0\} \rightarrow \mathbb{P}^{n-1}$ is a nonconstant $G$-invariant morphism, and indeed is a categorical quotient for the action of $\mathbb{C}^{*}$ on $\mathbb{C}^{n} \backslash\{0\}$. Thus in Example 3.2.4 above we have $\mathbb{P}^{n} / / L_{-} \mathbb{C}^{*}=\mathbb{P}^{n-1}$ while $\mathbb{P}^{n} / / L_{+} \mathbb{C}^{*}$ is empty and $\mathbb{P}^{n} / / L_{0} \mathbb{C}^{*}$ is a point.

Similarly, although following Mumford [48] we can define stable and semistable points for any linear action of a reductive group $G$ on a quasi-projective variety $X$, it is not necessarily the case that $U^{s s}=U \cap X^{s s}$ or that $U^{s}=U \cap X^{s}$ whenever $U$ is a $G$-invariant open subset of $X$. 
Definition 3.2.6. Let $X$ be a quasi-projective variety with an action of a reductive group $G$ and linearization $L$ on $X$. Then $y \in X$ is semistable for this linear action if there exists some $m \geq 0$ and $f \in H^{0}\left(X, L^{\otimes m}\right)^{G}$ not vanishing at $y$ such that the open subset

$$
X_{f}:=\{x \in X \mid f(x) \neq 0\}
$$

is affine, and $y$ is stable if in addition the action of $G$ on $X_{f}$ is closed with all stabilizers of dimension 0 .

Remark 3.2.7. Note that the line bundle $L$ is not required to be ample here. When $X$ is projective and $L$ is ample and $f \in H^{0}\left(X, L^{\otimes m}\right)^{G} \backslash\{0\}$ for some $m \geq 0$, then $X_{f}$ is affine if and only if $f$ is nonconstant or equivalently $m>0$. Thus Definition 3.2.6 agrees with $\S 3.2 .1$ for projective $X$ and ample $L$. Moreover when $X \subseteq \mathbb{A}^{n} \subseteq \mathbb{P}^{n}$ is affine and the linear $G$-action is given by a representation of $G$ in $G L(n ; k)$ embedded in $G L(n+1 ; k)$ in the usual way by taking a direct sum with a one-dimensional trivial representation of $G$, then we have

$$
X^{s s}=X
$$

and $X / / G=\operatorname{Spec}\left(k[X]^{G}\right.$ ) is a categorical quotient of $X$ (cf. Theorem 3.2.9 below), while $x \in X$ is stable if and only if there is some $f \in k[X]^{G}$ such that the action of $G$ on $X_{f}$ is closed with all stabilizers having dimension 0 .

Remark 3.2.8. One can see from these definitions that three things can go wrong in relating stable and semistable points for open immersions $U \subseteq X$ : (1) invariants do not necessarily extend, and even if an invariant $f$ extends then (2) affineness of $X_{f}$ is neither a necessary nor a sufficient condition for $U_{f}$ to be affine, and (3) the action on $U_{f}$ may be closed with all stabilizers of dimension 0 without the same being true of $X_{f}$.

Theorem 3.2.9 (Mumford). [48, Theorem 1.10] Let $X$ be an algebraic scheme over $k$ with an action of a reductive group $G$ and linearization $L$ on $X$. Then $X^{s s}$ has a quasi-projective categorical quotient $\phi: X^{s s} \rightarrow X / / G$ which restricts to a geometric quotient $\phi: X^{s} \rightarrow \phi\left(X^{s}\right)$ of $X^{s}$, where $\phi\left(X^{s}\right)=X^{s} / G$ is an open subset of $X / / G$.

Remark 3.2.10. The maximality property of $X^{s}$ described in Remark 3.2.2 extends to nonsingular quasi-projective varieties $X$.

\section{Generalizing GiT: Intrinsic Viewpoints}

Throughout, unless otherwise stated, $X$ will be a projective variety endowed with an ample line bundle $L$, on which an affine algebraic group $H$ acts linearly. As discussed previously, $H$ may be assumed to be connected unipotent. 
4.1. Global approaches. This subsection will be fairly informal, serving three purposes - to set the stage as naively as possible, to present some definitions, and to give a brief survey of some of the literature.

The inclusion $k[X]^{H} \hookrightarrow k[X]$ induces a rational "quotient" map of schemes $q: X \rightarrow \operatorname{Proj}\left(k[X]^{H}\right)$. The image of $q$ is a constructible subset, i.e. a finite union of locally closed subschemes. The goal is to understand $q$ well enough to construct canonically associated "quotient" varieties. There are a couple of interrelated approaches to analyzing $q$ and possible quotients:

- take large enough finitely generated approximations to $k[X]^{H}$, and

- understand $q$ restricted to open sets on which the action is nice.

For simplicity of notation we shall assume that the generic stabilizer has dimension 0 ; the discussion in general is not significantly different.

4.1.1. Naive stability. As in reductive GIT for projective varieties with ample line bundles, we might think of the semistable points as the points where some $H$-invariant does not vanish; that is, the points in the domain of definition of $q$.

Definition 4.1.1. A point $x \in X$ is naively semistable if $q(x)$ is well-defined. The set of naively semistable points $X^{n s s}$ is the domain of definition of $q$.

Consider an increasing filtration of $A=k[X]^{H}$ by finitely generated subrings $A_{i}$ with $\lim _{\rightarrow}\left(A_{i}\right)=A$. The inclusions $A_{i} \rightarrow A_{i+1} \rightarrow \cdots \rightarrow A \rightarrow k[X]$ induce rational maps

$$
q_{i}: X \rightarrow \operatorname{Proj}(A) \rightarrow \cdots \rightarrow \operatorname{Proj}\left(A_{i+1}\right) \rightarrow \operatorname{Proj}\left(A_{i}\right) .
$$

Because $X$ is Noetherian, the unstable set $X \backslash X^{n s s}$ is cut out by finitely many invariants, so for large enough $i$, the domain of definition of $q_{i}$ is $X^{n s s}$. Furthermore, when $i$ is large enough, $A_{i}$ contains a finite separating set of invariants; the natural rational map $q_{i+1, i}: \operatorname{Proj}\left(k[X]_{i+1}^{H}\right) \rightarrow \operatorname{Proj}\left(k[X]_{i}^{H}\right)$ then maps $q_{i+1}\left(X^{n s s}\right)$ bijectively onto $q_{i}\left(X^{n s s}\right)$.

It therefore makes sense to look for open subsets $U$ of $X^{\text {nss }}$ whose images $q_{i}(U)$ are varieties independent (up to isomorphism) of $i$, once $i$ is sufficiently large. Upper semi-continuity of $q_{i}$ for large $i$ determines a finite filtration of $X^{n s s}$ by opens $U_{j}$ indexed according to the maximal dimension $j \geq \operatorname{dim}(H)$ of components of the fiber at a point; provided that $A_{i}$ contains a separating set of invariants this filtration is independent of $i$. Restricting one's attention to the smallest of these open subvarieties, $U_{\operatorname{dim}(H)}$, is a good way to find analogs of "stable" points, since $q$ can only be an orbit map (and hence possibly a geometric quotient) on subsets of $U_{\operatorname{dim}(H)}$. 
Definition 4.1.2. Let $X$ be a projective variety with a linear $H$ action. A point $x \in X^{n s s}$ is said to be almost stable if its stabilizer group is trivial and $\operatorname{dim}\left(q^{-1}(q(x))\right)=\operatorname{dim}(H)$. The set of almost stable points we denote by $X^{a s}$.

Remark 4.1.3. If $k[X]^{H}$ is finitely generated and $\operatorname{Proj}\left(k[X]^{H}\right)$ is normal, then by standard results (see [5, Proposition 18.4]) the morphism $q$ restricted to $X^{\text {as }}$ is an open mapping, so its image is a variety. Restricting $q$ further to $U=q^{-1}(V)$ where $V$ is the union of open subsets of $q\left(X^{a s}\right)$ over which all geometric fibres are single orbits - what might be called the "naively stable" set - it follows [5, Proposition 6.6] that we obtain a geometric quotient (in fact this holds in general, see Remark 4.2.5).

Remark 4.1.4. Fauntleroy [17] considered an analog of this concept of almost stability: for $Y$ quasi-affine and normal on which a connected unipotent group $H$ acts with finite stabilizers, and $p: Y \rightarrow \operatorname{Spec}\left(k[Y]^{H}\right)$ the canonical morphism, if $\operatorname{dim}\left(p^{-1}(p(y))\right)=\operatorname{dim}(H)$ then he calls $y$ "semistable" for the action of $H$. He shows that $p$ restricted to the open subvariety of $Y$ consisting of these semistable points is a categorical quotient (in the category of varieties). Fauntleroy's results applied to open subvarieties of an affine cone over a normal projective variety $X$ with a linear $H$-action show that $q\left(X^{a s}\right)$ is a variety.

Another option is to find "quotient" varieties $Q$, obtained by throwing out selected closed $H$-invariant subvarieties of $\operatorname{Proj}\left(A_{i}\right)$ for sufficiently large $i$, which are as close as possible to the image of $q_{i}$ - ideally while retaining some other good feature, such as $k[X]^{H}=k[Q]$. To that end, removing those (finitely many) codimension 1 divisors in $\operatorname{Proj}\left(A_{i}\right)$ which contain dense open subsets disjoint from $i m\left(q_{i}\right)$ is a reasonable approach, although some points of $i m\left(q_{i}\right)$ are typically removed in the process and some points not in $\operatorname{im}\left(q_{i}\right)$ may remain: this is, very roughly, what we do later with strong reductive envelopes (see Definition 5.2.7 and, with more stringent conditions, Theorem 5.3.5). In any such construction, whether the quotient variety is suitably canonical becomes a serious issue.

Remark 4.1.5. In the case when $X$ is affine, Winkelmann [60] showed there exists a quasi-affine "quotient" variety $Q$ which admits a rational map from $X$ and such that $k[Q]=k[X]^{H}$. The idea here, too, is removal of codimension 1 divisors in $\operatorname{Spec}\left(A_{i}\right)$ for large enough $i$.

4.1.2. Quotients by free actions. One can also make use of auxiliary properties of a group action which ensure the existence of a well-behaved quotient; specifically, recall that in reductive GIT the action on the stable locus is proper. In the case of a connected unipotent group over a characteristic 0 field, the proper actions (being also set-theoretically free) are exactly [13, $\S 6.3$ Lemma 8] the schemetheoretically free actions [48, Definition 0.8]. By Artin [3] and Kollár [41], proper actions admit geometric quotients in the category of algebraic spaces. So by considering only those open subvarieties on which the action is proper, coupled 
with some condition that ensures the quotient is a variety, one obtains a notion of stable sets, though finding a canonical choice of stable set is an issue here. Fauntleroy [16] defines a notion of properly stable actions of connected unipotent groups on quasi-affine normal varieties over algebraically closed fields, which yield geometric quotients in the category of varieties. Conversely, sufficiently well behaved geometric quotients come from properly stable actions.

Definition 4.1.6. A linear action of a connected unipotent group $H$ on a normal quasi-affine variety $U$ is properly stable if the $H$-action on $U$ is proper and every $x \in U$ has a $H$-invariant open neighbourhood $W$ such that the nonempty fibres of the natural map of schemes $q: W \rightarrow \operatorname{Spec}\left(k[W]^{H}\right)$ are $H$-orbits.

Proposition 4.1.7 ([16]). Let $U$ be a normal quasi-affine variety over the algebraically closed field $k$, on which the connected unipotent group $H$ acts. Assume that the action of $H$ on $U$ is properly stable. Then the quotient map $q: U \rightarrow q(U) \subseteq \operatorname{Spec}\left(k[U]^{H}\right)$ is affine and a principal $H$-bundle (i.e. a locally trivial geometric quotient by [48, Proposition 0.9]). Conversely, if $Y$ is a variety and $q: U \rightarrow Y$ is a principal $H$-bundle, then the action of $H$ on $U$ is properly stable.

Remark 4.1.8. Note that $H$ is a special group, in the sense of Serre and Grothendieck, so all principal $H$-bundles are in fact Zariski, not just étale, locally trivial [52, $\S 2.6]$.

Remark 4.1.9. Unfortunately, a maximal properly stable open subset of an affine variety $X$ is not in general unique, as Example 4.1.10 below shows, though when $k[X]$ is a UFD then being properly stable turns out to be a local property, so the union of all properly stable open subsets is the unique maximal properly stable open subset [16, Thm 2.4]. By [27, II Prop 6.2, II Cor 6.16] when $X$ is an affine variety the condition that $k[X]$ be a UFD is the same as $\operatorname{Pic}(X)$ being trivial. When working with a projective variety $X$ the analogous condition is $\operatorname{Pic}(X)=\mathbb{Z}$.

Example 4.1.10. Let $H=\mathbb{C}^{+}$act on $\mathbb{P}^{3}$ via

$$
t\left[x_{0}: x_{1}: x_{2}: x_{3}\right]=\left[x_{0}: x_{1}: x_{2}+t x_{1}: x_{3}+t\left(2 x_{2}+x_{0}\right)+t^{2} x_{1}\right]
$$

and let $X$ be the smooth irreducible surface

$$
x_{1} x_{3}-x_{2}^{2}-x_{0} x_{2}=0 .
$$

Then $\mathbb{C}^{+}$acts set-theoretically freely but not properly on the affine variety $W=$ $X \cap \mathbb{A}^{3}$ where $\mathbb{A}^{3} \subseteq \mathbb{P}^{3}$ is defined by $x_{0} \neq 0$, and the (non-separated) quotient is the affine line $\mathbb{A}^{1}$ with a doubled point; $W$ is the union of two invariant open subsets on each of which the action is properly stable with quotient $\mathbb{A}^{1}$ (see [18]; cf. [10] for a similar example). Here points of an open subset of $X$ on which the $H$-action is properly stable are not necessarily naively stable in the sense of Definition 4.2.1. 
4.2. Gluing local quotients $X_{f} / / H$. In reductive GIT, the set $X^{s}$ of $G$-stable points of $X$ is a union of affine invariant hypersurface complements $X_{f}$ on which the action is closed and orbits have maximal dimension, and the geometric quotient of $X^{s}$ can be constructed by patching together the corresponding affine varieties $\operatorname{Spec}\left(k\left[X_{f}\right]^{G}\right)$. Unipotent actions on affine varieties are always closed, but when a unipotent group $H$ acts linearly on a projective variety $X$ and $f$ is an invariant section there is no guarantee that $k\left[X_{f}\right]^{H}$ is a finitely generated $k$ algebra, that the natural quotient map $q$ from $X_{f}$ to $\operatorname{Spec}\left(k\left[X_{f}\right]^{H}\right)$ is surjective, or even that the image of $q$ is a variety.

However one knows that the field of invariant rational functions $k(X)^{H}$ is finitely generated (by a theorem of Rosenlicht, see [11, Theorem 6.2]), so there is an invariant open, $X_{f}$, for which $k\left[X_{f}\right]^{H}$ is finitely generated. When $g$ is another invariant then $X_{f} \cap X_{g}=X_{f g}$ has a finitely generated ring of invariants $k\left[X_{f}\right]^{H}\left[g^{-1}\right]$. We will see that taking the union of all such open affines $X_{f}$ for which the natural map $q: X_{f} \rightarrow \operatorname{Spec}\left(k\left[X_{f}\right]^{H}\right)$ has sufficiently good properties, and patching the associated maps $q$, yields canonical open sets with nice quotients. Patching works here, in contrast with Example 4.1.10, since any orbit in $X_{f}$ is distinguished from any orbit in the complement of $X_{f}$ by the invariant $f$ itself.

Since we would like a stable set to have a geometric quotient, it is natural to impose the condition that $q: X_{f} \rightarrow \operatorname{Spec}\left(k\left[X_{f}\right]^{H}\right)$ should be a geometric quotient.

Definition 4.2.1. Let $X$ be a projective variety and let $H$ be a connected unipotent group acting linearly on $X$ with respect to an ample line bundle $L$. The set of naively stable points of $X$ (with respect to the linearization $L$ ) is

$$
X^{n s}=\bigcup_{f \in I^{n s}} X_{f}
$$

where

$$
\begin{gathered}
I^{n s}=\left\{f \in H^{0}\left(X, L^{\otimes m}\right)^{H} \text { for some } m>0 \mid k\left[X_{f}\right]^{H}\right. \text { is finitely generated, and } \\
\left.q: X_{f} \longrightarrow \operatorname{Spec}\left(k\left[X_{f}\right]^{H}\right) \text { is a geometric quotient }\right\} .
\end{gathered}
$$

Proposition 4.2.2. $q\left(X^{n s}\right)$ is a quasi-projective variety and $q: X^{n s} \rightarrow q\left(X^{n s}\right)$ is a geometric quotient.

Proof. If $f \in I^{n s}$ then $q\left(X_{f}\right)=\operatorname{Spec}\left(k\left[X_{f}\right]^{H}\right)$ is an affine variety. By the Noetherian property we can choose $f_{0}, \ldots, f_{r} \in I^{n s}$ such that $X^{n s}=\bigcup_{j=0}^{r} X_{f_{j}}$, and without loss of generality we can assume that there is some $m>0$ such that $f_{j} \in H^{0}\left(X, L^{\otimes m}\right)^{H}$ for $0 \leq j \leq r$. For each $j$ by the definition of $I^{n s}$ we can choose finitely many generators $\left\{f_{i j}: 1 \leq i \leq q_{j}\right\}$ for $k\left[X_{f_{j}}\right]^{H}$, which we can express as $f_{i j}=g_{i j} /\left(f_{j}\right)^{\ell}$ with $g_{i j} \in H^{0}\left(X, L^{\otimes \ell m}\right)^{H}$ for some large $\ell>0$. 
Now let $M=r+\sum_{j=0}^{r} q_{j}$ and define $s: X^{n s} \rightarrow \mathbb{P}^{M}$ to have homogeneous coordinates given by the sections $f_{j}^{\ell}$ and $g_{i j}$ (for $0 \leq j \leq r$ and $1 \leq i \leq q_{j}$ ) of $L^{\otimes \ell m}$. If $y_{0}, \ldots, y_{r}$ are the first $r+1$ homogeneous coordinates on $\mathbb{P}^{M}$ which pull back to $f_{0}^{\ell}, \ldots, f_{r}^{\ell}$ on $X^{n s}$, then $s$ maps $X^{n s}$ into $\bigcup_{j=0}^{r}\left(\mathbb{P}^{M}\right)_{y_{j}}$ and for $0 \leq j_{0} \leq r$ we have

$$
s^{-1}\left(\left(\mathbb{P}^{M}\right)_{y_{j_{0}}}\right)=X_{f_{j_{0}}}
$$

where

$$
s: X_{f_{j_{0}}} \rightarrow\left(\mathbb{P}^{M}\right)_{y_{j_{0}}} \cong \mathbb{A}^{M}
$$

is the composition of $q: X_{f_{j_{0}}} \rightarrow q\left(X_{f_{j_{0}}}\right)=\operatorname{Spec}\left(k\left[X_{f_{j_{0}}}\right]^{H}\right)$ with the embedding of $\operatorname{Spec}\left(k\left[X_{f_{0}}\right]^{H}\right)$ as a closed subvariety of $\mathbb{A}^{M}$ defined by the subset

$$
\left\{\frac{f_{0}^{\ell}}{f_{j_{0}}^{\ell}}, \ldots, \frac{f_{0}^{\ell}}{f_{j_{0}}^{\ell}}\right\} \cup\left\{\frac{g_{i j}}{f_{j_{0}}^{\ell}}: 0 \leq j \leq r, 1 \leq i \leq q_{j}\right\}
$$

of $k\left[X_{f_{j_{0}}}\right]^{H}$ which includes the generators

$$
\left\{f_{i j}=\frac{g_{i j}}{f_{j_{0}}^{\ell}}: 0 \leq i \leq q_{j_{0}}\right\}
$$

of $k\left[X_{f_{j_{0}}}\right]^{H}$. It follows that $s$ is the composition of $q: X^{n s} \rightarrow q\left(X^{n s}\right)=$ $\bigcup_{j=0}^{r} q\left(X_{f_{j}}\right)$ with an embedding of $q\left(X^{n s}\right)$ as a locally closed subvariety of $\mathbb{P}^{M}$, and moreover that $q: X^{n s} \rightarrow q\left(X^{n s}\right)$ is a geometric quotient since $q: X_{f_{j}} \rightarrow$ $q\left(X_{f_{j}}\right)$ is a geometric quotient for $0 \leq j \leq r$ by the definition of $I^{n s}$.

We can also consider a more stringent condition, that $q: X_{f} \longrightarrow \operatorname{Spec}\left(k\left[X_{f}\right]^{H}\right)$ be a locally trivial geometric quotient (that is, an $H$-principal bundle with base $\left.\operatorname{Spec}\left(k\left[X_{f}\right]^{H}\right)\right)$.

Definition 4.2.3. The set of locally trivial stable points is

$$
X^{l t s}=\bigcup_{f \in I^{l t s}} X_{f}
$$

where

$$
\begin{array}{r}
I^{l t s}=\left\{f \in H^{0}\left(X, L^{\otimes m}\right)^{H} \text { for some } m>0 \mid k\left[X_{f}\right]^{H}\right. \text { is finitely generated, and } \\
\left.q: X_{f} \longrightarrow \operatorname{Spec}\left(k\left[X_{f}\right]^{H}\right) \text { is a locally trivial geometric quotient }\right\}
\end{array}
$$

Then $X^{\text {lts }}$ is an open subset of $X^{n s}$, and by patching together the natural maps $q: X_{f} \rightarrow \operatorname{Spec}\left(k\left[X_{f}\right]^{H}\right)$ as in Proposition 4.2 .2 we obtain a locally trivial geometric quotient $q: X^{\text {lts }} \rightarrow q\left(X^{\text {lts }}\right)$ where $q\left(X^{l t s}\right) \cong X^{l t s} / H$ is an open subvariety of $q\left(X^{n s}\right) \cong X^{n s} / H$. 
Remark 4.2.4. Note that for a reductive group acting with trivial isotropy on an invariant open subvariety $X_{f}$ local triviality is not a more stringent condition; that is, $X^{l t s}=X^{n s}$. Indeed the quotient map is affine, and so by Mumford [48, Proposition 0.8$]$ it is proper, and so here is scheme-theoretically free $[13, \S 6.3$ Lemma 8]; then by [48, Proposition 0.9] it is a principal bundle.

Remark 4.2.5. Another option is to ignore the finitely generated condition on $k\left[X_{f}\right]^{H}$ altogether, and work with $\operatorname{Spec}\left(k[X]^{H}\right)$ and $\operatorname{Spec}\left(k\left[X_{f}\right]^{H}\right)$ as schemes and the natural map $q: X_{f} \rightarrow \operatorname{Spec}\left(k\left[X_{f}\right]^{H}\right) \subseteq \operatorname{Spec}\left(k[X]^{H}\right)$ as a map of schemes. This is the approach taken by Greuel and Pfister [21], coinciding with that of Dixmier and Raynaud [10]. For stability they require less than Definition 4.2.1; they demand that the image $q\left(X_{f}\right)$ should be an open subscheme of $\operatorname{Spec}\left(k[X]^{H}\right)$ with $q: X_{f} \rightarrow q\left(X_{f}\right)$ an open map whose geometric fibres are just the $H$-orbits in $X_{f}$. (Strictly speaking their definition is for affine varieties, or quasi-affine with a choice of affine embedding). However they show, using [10, Proposition 2.2.2], that the open subscheme $q\left(X_{f}\right)$ of $\operatorname{Spec}\left(k[X]^{H}\right)$ is in fact then a variety, and furthermore that the restriction of the natural map of schemes $q: X \longrightarrow$ $\operatorname{Spec}\left(k[X]^{H}\right)$ to their stable set onto its image in $\operatorname{Spec}\left(k[X]^{H}\right)$ is a geometric quotient in the category of varieties. It follows that their definition of stability is essentially equivalent to $X^{n s}$.

In a similar manner, we can mimic the GIT construction of $X^{s s}$ and its categorical quotient by patching together the affine varieties $\operatorname{Spec}\left(k\left[X_{f}\right]^{H}\right)$ for all those open subvarieties $X_{f}$ with finitely generated rings of $H$-invariants. We continue to assume that $X$ is a projective variety and that $H$ is a connected unipotent group acting linearly on $X$ with respect to an ample line bundle $L$.

Definition 4.2.6. We define the finitely generated semistable set by

$$
X^{s s, f g}=\bigcup_{f \in I^{s s, f g}} X_{f}
$$

where

$$
I^{s s, f g}=\left\{f \in H^{0}\left(X, L^{\otimes m}\right)^{H} \text { for some } m>0 \mid k\left[X_{f}\right]^{H} \text { is finitely generated }\right\} .
$$

It is not difficult to check using the proof of Proposition 4.2.2 that the affine varieties $\operatorname{Spec}\left(k\left[X_{f}\right]^{H}\right)$ for $f \in I^{s s, f g}$ patch to give a quasi-projective variety which is an open subscheme of the scheme $\operatorname{Proj}\left(k[X]^{H}\right)$; see Proposition 4.2.9 below. We introduce some terminology for such quotients:

Definition 4.2.7. Let $q: X^{s s, f g} \rightarrow \operatorname{Proj}\left(k[X]^{H}\right)$ be the natural morphism of schemes. Then the enveloped quotient of $X^{s s, f g}$ is $q: X^{s s, f g} \rightarrow q\left(X^{s s, f g}\right)$, where $q\left(X^{s s, f g}\right)$ is a dense constructible subset of the enveloping quotient

$$
X / / H=\bigcup_{f \in I^{s s, f g}} \operatorname{Spec}\left(k\left[X_{f}\right]^{H}\right)
$$


of $X^{s s, f g}$.

Remark 4.2.8. The morphism $q: X^{s s, f g} \rightarrow X / / H$ is not in general surjective, even when $k[X]^{H}$ is finitely generated, as we will see in $\S 6$. The enveloping quotient $X / / H$ is thus not in general a categorical quotient of $X^{s s, f g}$.

Proposition 4.2.9. The enveloping quotient $X / / H$ is a quasi-projective variety with an ample line bundle $L_{H} \rightarrow X / / H$ which pulls back to a positive tensor power of $L$ under the natural map $q: X^{s s, f g} \rightarrow X / / H$.

Proof. This follows from a trivial modification of the proof of Proposition 4.2.2, replacing $I^{n s}$ with $I^{s s, f g}$, replacing $q\left(X^{n s}\right)$ with $X / / H$ and replacing $q\left(X_{f}\right)$ with $\operatorname{Spec}\left(k\left[X_{f}\right]^{H}\right)$ for any invariant $f$ throughout.

Proposition 4.2.10. If $k[X]^{H}$ is finitely generated then $X / / H$ is the projective variety $\operatorname{Proj}\left(k[X]^{H}\right)$.

Proof. When $k[X]^{H}$ is finitely generated then so is $k\left[X_{f}\right]^{H}$ for any $f \in I=$ $\bigcup_{m>0} H^{0}\left(X, L^{\otimes m}\right)^{H}$, so $I^{s s, f g}=I$ and hence

$$
X / / H=\bigcup_{f \in I} \operatorname{Spec}\left(k\left[X_{f}\right]^{H}\right)=\operatorname{Proj}\left(k[X]^{H}\right) .
$$

Lemma 4.2.11. If $X$ is normal, then $X^{a s} \subset X^{s s, f g}$.

Proof. By definition $X^{a s} \subseteq X^{n s s}=\bigcup_{f \in I} X_{f}$ where $I=\bigcup_{m>0} H^{0}\left(X, L^{\otimes m}\right)^{H}$. If $f \in I$ then $X_{f}$ is affine, so we know by [17] (where normality is assumed) that $q\left(X^{a s} \cap X_{f}\right)$ is an open subvariety of $\operatorname{Spec}\left(k\left[X_{f}\right]^{H}\right)$. Thus it is the complement of a closed subscheme cut out by functions $f_{i} \in k\left[X_{f}\right]^{H}$ which we can assume are of the form $f_{i}=g_{i} / f^{\ell}$ for some large $\ell \geq 0$ where $g_{i} \in k[X]^{H}$, and hence is a union of affine schemes $\operatorname{Spec}\left(k\left[X_{f}\right]^{H}\right)_{f_{i}}=\operatorname{Spec}\left(k\left[X_{g_{i} f}\right]^{H}\right)$; as subschemes of the variety $q\left(X^{a s} \cap X_{f}\right)$ these are themselves affine varieties, and hence each $k\left[X_{g_{i} f}\right]^{H}$ is finitely generated. But then $X^{a s}$ is the union of the corresponding opens $X_{g_{i} f}=q^{-1}\left(\operatorname{Spec}\left(k\left[X_{g_{i} f}\right]^{H}\right)\right.$ of $X$, and so $X^{a s} \subseteq X^{s s, f g}$ as required.

\section{Generalizing Git: Induced Reductive aCtions - From $H$ to $G$}

5.1. Stability for $G \times_{H} X$. A couple of fundamental results on algebraic homogeneous spaces will be useful (see [52, Theorem 4.17] and [25, Corollary 2.8], respectively).

Proposition 5.1.1 (Matsushima). Given a reductive $G$, then $G / H$ is affine if and only if $H$ is reductive. 
Proposition 5.1.2. If $H$ is unipotent, then $H$ is an observable subgroup of $G$; that is, $G / H$ is a quasi-affine variety.

Corollary 5.1.3. If $H$ is a positive dimensional unipotent group and $G$ is reductive, then $G / H$ is quasi-affine but not affine.

Choose a reductive group $G$ with the connected unipotent group $H$ as a closed subgroup. A linearization of an $H$-action on a projective (or quasi-projective) $X$ with respect to an embedding of $X$ in $\mathbb{P}^{n}$ gives us a representation of $H$ in $S L(n+1 ; k)$; if we assume, as without loss of generality we may, that this representation is faithful, then we can always choose $G$ to be $S L(n+1 ; k)$. Let $G \times{ }_{H} X$ denote the quotient of $G \times X$ by the free action of $H$ defined by $h(g, x)=$ $\left(g h^{-1}, h x\right)$. There is an induced $G$-action on $G \times_{H} X$ where $G$ acts on itself by left multiplication.

Remark 5.1.4. With respect to the natural $G$-equivariant projection to the homogeneous space $G / H$ given by $[g, x] \mapsto g H$, the algebraic space $G \times_{H} X$ is a fiber bundle with fibers isomorphic to $X$ presented as sets of the form $g X$; it is not merely an algebraic space, but is in fact an algebraic variety [52][Theorem 4.19].

If the action of $H$ on $X$ extends to $G$ we have an isomorphism of $G$-varieties $G \times{ }_{H} X \cong(G / H) \times X$ given by

$$
[g, x] \mapsto(g H, g x) .
$$

When $X$ is affine we have

$$
k\left[G \times_{H} X\right]^{G} \cong k[X]^{H} .
$$

When $X$ is projective (or more generally quasi-projective) and $L \rightarrow X$ is a very ample $H$-linearization inducing an embedding of $X$ in $\mathbb{P}^{n}$, and $G$ is a subgroup of $S L(n+1 ; k)$, then we get a very ample $G$-linearization on $G \times_{H} X$ by pulling back $\mathcal{O}_{\mathbb{P}^{n}}(1)$ via the sequence:

$$
G \times_{H} X \hookrightarrow G \times_{H} \mathbb{P}^{n} \cong(G / H) \times \mathbb{P}^{n} \rightarrow \mathbb{P}^{n},
$$

taking the trivial bundle on the quasi-affine variety $G / H$; by choosing a $G$ equivariant embedding of $G / H$ in an affine space $\mathbb{A}^{m}$ with a linear $G$-action we get a $G$-equivariant embedding of $G \times_{H} X$ in

$$
\mathbb{A}^{m} \times \mathbb{P}^{n} \subset \mathbb{P}^{m} \times \mathbb{P}^{n} \subset \mathbb{P}^{n m+m+n}
$$

By abuse of notation let us also call this $G$-linearization $L$. Then

$$
k\left[G \times_{H} X\right]^{G}=\bigoplus_{m \geq 0} H^{0}\left(G \times_{H} X, L^{\otimes m}\right)^{G} \cong \bigoplus_{m \geq 0} H^{0}\left(X, L^{\otimes m}\right)^{H}=k[X]^{H} .
$$


Remark 5.1.5. If the action of $H$ on $X$ extends to $G$ we have $G \times_{H} X \cong(G / H) \times$ $X$ via the isomorphism (1) above, in which case this identification of rings of invariants is sometimes known as the Borel transfer principle (see [11, Lemma 4.1]).

Let $i: X \rightarrow G \times_{H} X$ be the closed immersion given by $x \mapsto[e, x]$.

Definition 5.1.6. Let the set of Mumford stable points for a linear $H$-action on a quasi-projective variety $X$ be $X^{m s}=i^{-1}\left(\left(G \times_{H} X\right)^{s}\right)$. Similarly, the set of Mumford semistable points for the linear $H$-action on $X$ is $X^{m s s}=i^{-1}\left(\left(G \times_{H}\right.\right.$ $\left.X)^{s s}\right)$. Here $\left(G \times_{H} X\right)^{s}$ and $\left(G \times_{H} X\right)^{s s}$ are defined as in Definition 3.2.6 for the induced linear action of $G$ on $G \times_{H} X$.

These sets admit geometric and categorical quotients, respectively, by standard reductive GIT. But because $H$ is unipotent something quite interesting happens.

Lemma 5.1.7. Every Mumford semistable point is Mumford stable; that is, $X^{m s}=X^{m s s}$.

Proof. Let $O$ be an $H$-orbit in $X^{m s s} \backslash X^{m s}$, so that $G \times_{H} O$ is a $G$-orbit in an affine hypersurface complement $\left(G \times_{H} X\right)_{F}$ which is either closed of nonmaximal dimension or not closed in $\left(G \times_{H} X\right)_{F}$. In each case there is a unique closed orbit $G \times_{H} O^{\prime}$ in the closure of $G \times_{H} O$ in $\left(G \times_{H} X\right)_{F}$, which is of nonmaximal dimension. In an affine variety a closed $G$-orbit is of course affine, so by Proposition 5.1.1 the corresponding stabilizer is a reductive subgroup of $G$. Hence any point of $G \times{ }_{H} O^{\prime}$ must have a positive dimensional reductive stabilizer, which is a contradiction since the stabilizer is a subgroup of $H$ and so is unipotent. Thus there are no Mumford semistable points which are not Mumford stable; that is, $X^{m s}=X^{m s s}$.

Remark 5.1.8. When coupled with the fact that $\left(G \times_{H} X\right)_{F}$ is always quasi-affine (see Corollary 5.2.9), it follows from this that whatever notion one comes up with for "strictly semistable" orbits that are not Mumford stable, such orbits must lie in quasi-affine but not affine $\left(G \times_{H} X\right)_{F}$. This is a useful geometric observation, to be used again in the main theorem below.

A priori the definitions of $X^{m s}$ and $X^{m s s}$ depend on the choice of the reductive group $G$, but in fact they do not.

Proposition 5.1.9. The open subsets $X^{m s}$ and $X^{m s s}$ of $X$ are intrinsically defined, in that they depend only on the linear $H$-action on $X$ and are independent of the choice of $G$.

Proof. By Lemma 5.1.7 it is enough to consider $X^{m s s}$. We may assume that both $H$ and $G$ are subgroups of the general linear group $G L(n+1 ; k)$ associated 
to the projective embedding of $X$ given by the linearization. Suppose that $G_{1}$ and $G_{2}$ are both subgroups of $G_{0}=G L(n+1 ; k)$ containing $H$. Let $f$ be an $H$-invariant, and let $F_{1}, F_{2}$ and $F_{0}$ be the associated $G_{1}, G_{2}$ and $G_{0}$ invariants on $G_{1} \times_{H} X, G_{2} \times_{H} X$, and $G_{0} \times_{H} X$, respectively. By definition of $X^{m s s}$, we want to show that $\left(G_{1} \times_{H} X\right)_{F_{1}}$ is affine if and only if $\left(G_{2} \times_{H} X\right)_{F_{2}}$ is affine. But then it suffices to show that $\left(G_{1} \times_{H} X\right)_{F_{1}}$ is affine if and only if $\left(G_{0} \times_{H} X\right)_{F_{0}}$ is affine.

If $\left(G_{1} \times_{H} X\right)_{F_{1}}$ is affine, then because $\left(\left(G_{1} \times_{H} X\right)_{F_{1}}\right) \times_{G_{1}} G_{0}=\left(\left(G_{1} \times_{H} X\right) \times_{G_{1}}\right.$ $\left.G_{0}\right)_{F_{0}}=\left(G_{0} \times_{H} X\right)_{F_{0}}$, and both $G_{1}$ and $G_{0}$ are affine, the latter is affine [52, p. 196]. Conversely, if $\left(G_{0} \times_{H} X\right)_{F_{0}}$ is affine, then so is any closed subvariety. But the inclusion of $\left(G_{1} \times_{H} X\right)_{F_{1}}$ in $\left(G_{0} \times_{H} X\right)_{F_{0}}$ is a closed immersion, so it must be an affine variety.

The intrinsic nature of $X^{m s}$ and $X^{m s s}$ can be seen more geometrically by the following characterization.

Proposition 5.1.10. The Mumford stable points are precisely the locally trivially stable points; that is, $X^{m s}=X^{l t s}$.

Proof. Consider $x \in X^{\text {lts }}$. Then $x \in X_{f}$ for $f$ an $H$-invariant section of a positive tensor power of $L$, where $\phi: X_{f} \rightarrow X_{f} / H$ is a principal $H$-bundle and $X_{f} / H=\operatorname{Spec}\left(k[X]^{H}\right)_{f}$ is an affine variety. By étale descent, $\phi$ is a principal $H$-bundle if and only if $\pi:\left(G \times_{H} X\right)_{F} \rightarrow X_{f} / H$ is a principal $G$-bundle, for $F$ the corresponding $G$-invariant. But by [48, Proposition 0.7] this means $\pi$ is an affine morphism, so $\left(G \times_{H} X\right)_{F}$ is affine. It follows that $x \in X^{m s}$.

In the reverse direction we see that $X^{m s} \subseteq X^{\text {lts }}$ as follows. $G$ acts schemetheoretically freely on $\left(G \times_{H} X\right)^{s}$ because the action is proper and set-theoretically free $[13, \S 6.3$ Lemma 8]. Furthermore by reductive GIT this action has a geometric quotient $\left(G \times_{H} X\right)^{s} \rightarrow\left(G \times_{H} X\right)^{s} / G \cong X^{m s} / H$. Hence by Mumford [48, Proposition 0.9] $\left(G \times{ }_{H} X\right)^{s} \rightarrow X^{m s} / H$ is a $G$-principal bundle, so by descent $X^{m s} \rightarrow X^{m s} / H$ is an $H$-principal bundle, and furthermore it is locally described by $X_{f} \rightarrow X_{f} / H$ for some collection $f$ of $H$-invariants.

Remark 5.1.11. It follows that any $H$-orbit in $X^{\text {ns }} \backslash X^{\text {lts }}$ (see Definitions 4.2.1 and 4.2.3) has the property that the corresponding $G$-orbit lies in a quasi-affine but not affine $\left(G \times_{H} X\right)_{F}$ (cf. Remark 5.1.8 above).

Remark 5.1.12. When it is convenient to do so we can choose $G$ to be semisimple throughout this section; indeed we can make a canonical choice of $G$ as $S L(n+$ $1 ; k)$, but it is often easier to work with a smaller semisimple or reductive group $G$.

5.2. Completions and reductive envelopes. The techniques of reductive GIT are most effective when applied to projective varieties, so it makes sense to choose 
a $G$-equivariant projective completion of $G \times_{H} X$ together with an extension of the $G$-linearization. However, as discussed in Section 3.2.2, GIT does not behave well with respect to $G$-equivariant open inclusions. Recalling from Lemma 5.1.7 that the $G$-semistable points of $G \times_{H} X$ are all $G$-stable, we may summarize the issues as follows:

(1) Some invariants may not extend to the boundary, so $G$-stable points of $G \times_{H} X$ could become unstable in $\overline{G \times{ }_{H} X}$.

(2) Even if a given invariant $F$ does extend from $G \times_{H} X$ to $\overline{G \times{ }_{H} X}$, the open subvariety $\left(G \times_{H} X\right)_{F}$ may be affine when $\left(\overline{G \times{ }_{H} X}\right)_{F}$ is not, so again $G$-stable points in $G \times_{H} X$ could become unstable in $\overline{G \times{ }_{H} X}$.

(3) Alternatively, if an invariant $F$ extends from $G \times_{H} X$ to $\overline{G \times{ }_{H} X}$, then $\left(\overline{G \times{ }_{H} X}\right)_{F}$ might be affine when $\left(G \times_{H} X\right)_{F}$ is not, so unstable points of $G \times_{H} X$ could become semistable in $\overline{G \times{ }_{H} X}$.

(4) If the $G$-action on $\left(G \times_{H} X\right)_{F}$ is closed the $G$-action on $\left(\overline{G \times_{H} X}\right)_{F}$ need not be, so $G$-stable points in $G \times_{H} X$ may become strictly semistable in $\overline{G \times{ }_{H} X}$.

Remark 5.2.1. Recall that if $L \rightarrow Y$ is an ample line bundle, $Y$ is projective, and $F$ is a section of $L$, then $Y_{F}$ is affine. So the second issue above is not a problem for ample extensions of the $G$-linearization. For ample extensions, it also follows that the third issue above can be refined since unstable points of $G \times_{H} X$ cannot become stable in $\overline{G \times{ }_{H} X}$ and hence can only be strictly semistable or unstable in $\overline{G \times{ }_{H} X}$ : it follows from Proposition 3.1.1 that if $O$ is a $G$-orbit in $G \times_{H} X$ whose points are stable in $\overline{G \times{ }_{H} X}$ then there is an invariant $F$ which is nonzero on $O$ and zero on the boundary, so that $\left(G \times_{H} X\right)_{F}=\left(\overline{G \times{ }_{H} X}\right)_{F}$ is affine; thus the points of $O$ are semistable (and indeed stable by Lemma 5.1.7) in $G \times_{H} X$.

The first task is to find a completion $\overline{G \times{ }_{H} X}$ together with an extension of the line bundle $L$, such that "sufficiently many" invariants $F_{i}$ extend over the boundary with $\overline{G \times}_{H} X_{F_{i}}$ affine.

Definition 5.2.2. Let $X$ be a quasi-projective variety with an $H$-action with linearization $L$, and let $G$ be any reductive group containing $H$. A finite separating set $S$ (in the sense of Definition 3.0.1) of invariant sections of positive tensor powers of $L$ is a finite fully separating set of invariants for the linear $H$-action on $X$ if

(i) for every $x \in X^{m s}$ there exists $f \in S$ with associated $G$-invariant $F$ over $G \times_{H} X$ such that $x \in\left(G \times_{H} X\right)_{F}$ and $\left(G \times_{H} X\right)_{F}$ is affine; and

(ii) for every $x \in X^{s s, f g}$ there exists $f \in S$ such that $x \in X_{f}$ and $S$ is a generating set for $k\left[X_{f}\right]^{H}$.

Remark 5.2.3. The proof of Proposition 5.1.9 shows that this definition is independent of the choice of $G$. 
We now make a sequence of definitions to address the issues enumerated above.

Definition 5.2.4. Let $X$ be a quasi-projective variety with a linear $H$-action with respect to an ample line bundle $L$ on $X$. Fix a closed immersion $H \hookrightarrow G$ for $G$ a reductive group, and fix a $G$-equivariant projective completion $\overline{G \times{ }_{H} X}$ of $G \times{ }_{H} X$ together with a $G$-linearization $L^{\prime}$ which restricts to the $H$-linearization $L$ on $X$. If every $H$-invariant $f$ in some finite fully separating set of invariants $S$ for the $H$-action on $X$ (in the sense of Definition 5.2.2) extends to a $G$-invariant section of a tensor power of $L^{\prime}$ over $\overline{G \times_{H} X}$, we will call $\overline{G \times_{H} X}$ together with the $G$-linearization $L^{\prime}$ a reductive envelope of the linear $H$-action on $X$ (with respect to the finite fully separating set of invariants $S$ ).

Definition 5.2.5. With notation as above, if there exists such an $S$ for which every $f \in S$ extends to a $G$-invariant section $F$ over $\overline{G \times{ }_{H} X}$ such that $\left(\overline{G \times{ }_{H} X}\right)_{F}$ is affine, then we say that $\left(\overline{G \times{ }_{H} X}, L^{\prime}\right)$ is a fine reductive envelope. If $L^{\prime}$ is ample then we say this is an ample reductive envelope.

Remark 5.2.6. Note that an ample reductive envelope is automatically fine.

Issue (4) above can be overcome when the following stringent condition holds, from which other good properties will also follow (see Theorem 5.3.5).

Definition 5.2.7. With the notation of Definition 5.2.4 and given a reductive envelope $\left(\overline{G \times{ }_{H} X}, L^{\prime}\right)$ with respect to a finite fully separating set of invariants $S$, let $D_{1}, \ldots, D_{r}$ denote the codimension 1 components of the boundary of $G \times_{H} X$ in $\overline{G \times{ }_{H} X}$. If every $f \in S$ extends to a $G$-invariant $F$ over $\overline{G \times{ }_{H} X}$ which vanishes on each component $D_{j}$, then $\left(\overline{G \times{ }_{H} X}, L^{\prime}\right)$ will be called a strong reductive envelope of the linear $H$-action on $X$.

Proposition 5.2.8. Given a faithful $H$-action on a quasi-projective variety $X$ with an ample linearization $L$, there always exists an integer $m>0$ and an ample reductive envelope for the induced linearization on $L^{\otimes m}$.

Proof. The linearization provides an embedding of $X$ into a projective space such that $H$ acts as a subgroup of the associated general linear group; without loss of generality $L$ is the hyperplane line bundle on this projective space. By the Noetherian property a finite fully separating set of invariants $S$ exists. Moreover there is some $M>0$ such that there is a finite fully separating set $S$ for which every $f \in S$ is an invariant section of $L^{\otimes M}$. Take the union of $S$ and a basis for those sections of $L^{\otimes M}$ which extend over the projective space in which $X$ is embedded. This set of sections defines an embedding of $X$ into some larger projective space $\mathbb{P}^{n}$ such that $H$ acts as a subgroup of $G=G L(n+1 ; k)$ and moreover every invariant in $S$ extends to an $H$-invariant on $\mathbb{P}^{n}$. As in Remark 5.1.5, since the action of $H$ on $\mathbb{P}^{n}$ extends to $G$ we have $G \times{ }_{H} \mathbb{P}^{n} \cong(G / H) \times \mathbb{P}^{n}$, so that if $\overline{G / H}$ is any $G$-equivariant projective completion of $G / H$ then we can 
identify $G \times{ }_{H} \mathbb{P}^{n}$ with an open subvariety of $\overline{G / H} \times \mathbb{P}^{n}$ and hence obtain a $G$-equivariant projective embedding of $G \times{ }_{H} \mathbb{P}^{n}$. Every $f \in S$ extends to an $H$-invariant on $\mathbb{P}^{n}$ and hence to a $G$-invariant on $G \times_{H} \mathbb{P}^{n}$, so by using the construction above we can find a modified $G$-equivariant projective embedding of $G \times{ }_{H} \mathbb{P}^{n}$ such that every $f \in S$ extends to a $G$-invariant section of the hyperplane line bundle on the ambient projective space $\mathbb{P}^{N}$. Then the closure of $G \times_{H} X$ in $\mathbb{P}^{N}$ together with the restriction of $\mathcal{O}_{\mathbb{P}^{N}}(1)$ to $\overline{G \times{ }_{H} X}$ is an ample reductive envelope for some positive power of the linearization $L$.

Corollary 5.2.9. Let $f$ be an $H$-invariant of $(X, L)$, and $F$ be the corresponding $G$ invariant over $G \times_{H} X$. Then $\left(G \times_{H} X\right)_{F}$ is a quasi-affine variety.

Proof. Any $F$ may be included in the set of invariants from the proof of Proposition 5.2.8. Because the result is an ample reductive envelope, $(\overline{G \times H X})_{F}$ is affine.

Remark 5.2.10. We will see in Remark 5.3.11 that smooth varieties $X$ do not always have smooth ample reductive envelopes; indeed, we cannot necessarily find ample reductive envelopes satisfying a weaker condition than nonsingularity which we will call gentleness (see Definition 5.3.7 below). The heart of the matter is contained in the combination of Proposition 5.3.9 below with the observation that if a normal affine variety $X$ has a fine strong reductive envelope $\left(\overline{G \times{ }_{H} X}, L^{\prime}\right)$ with respect to a finite fully separating set of invariants $S$ which includes a nonzero constant function on $X$, then $k[X]^{H}$ is finitely generated. To see this, first note that without loss of generality, one may take a normalization of the reductive envelope. Let $f \in S$ be a nonzero constant function on $X$ and $F$ denote its associated $G$-invariant on $\overline{G \times{ }_{H} X}$. Then the affine variety $\left(\overline{G \times_{H} X}\right)_{F}$ contains $\left(G \times_{H} X\right)_{F}$ as a codimension 2 complement, so by normality $G$-invariant sections over the latter canonically extend to sections over the former. Because $G$ is reductive, we know the space of $G$-invariant sections over the affine variety $\left(\overline{G \times{ }_{H} X}\right)_{F}$ is finitely generated. But $\left(G \times_{H} X\right)_{F}=G \times_{H} X$ since $F$ restricts to a nonzero constant function over $X$, and $G$-invariant sections over $G \times_{H} X$ correspond exactly to $H$-invariant sections over $X$.

Definition 5.2.11. Let $X$ be a projective variety with a linear $H$-action and a reductive envelope $\overline{G \times{ }_{H} X}$ in the sense of Definition 5.2.4. Label the inclusions $i: X \hookrightarrow G \times_{H} X$ and $j: G \times_{H} X \hookrightarrow \overline{G \times_{H} X}$. Let the completely stable points of $X$ with respect to the reductive envelope be the set

$$
X^{\bar{s}}=(j \circ i)^{-1}\left(\overline{G \times{ }_{H} X^{s}}\right) .
$$

Let the set of completely semistable points be

$$
X^{\overline{s s}}=(j \circ i)^{-1}\left(\overline{G \times H}^{s s}\right) .
$$


Remark 5.2.12. It will follow from Theorems 5.3.1 and 5.3.5 that if $\overline{G \times_{H} X}$ is normal and together with $G$-linearization $L^{\prime}$ forms a fine strong reductive envelope, then $X^{\bar{s}}$ and $X^{\overline{s s}}$ are independent of the choice of $\overline{G \times{ }_{H} X}$ and $L^{\prime}$.

5.3. Main Theorem and Corollaries. As in Definition 5.2.11 let $\overline{G \times{ }_{H} X}$ be a reductive envelope for a projective variety $X$ with a linear $H$-action, and let

$$
i: X \hookrightarrow G \times_{H} X \text { and } j: G \times_{H} X \hookrightarrow \overline{G \times_{H} X}
$$

denote the natural injections. Throughout

$$
\pi:\left(\overline{G \times \times_{H} X}\right)^{s s} \rightarrow \overline{G \times \times_{H} X} / / G
$$

will denote the GIT quotient map for the reductive envelope, restricted to open subsets (by abuse of notation) as appropriate.

Theorem 5.3.1. Let $X$ be a normal projective variety with a linear $H$-action, for $H$ a connected unipotent group, and let $\left(\overline{G \times{ }_{H} X}, L^{\prime}\right)$ be any fine reductive envelope as in Definition 5.2.5. Then

$$
X^{\bar{s}} \subseteq X^{l t s}=X^{m s}=X^{m s s} \subseteq X^{n s} \subseteq X^{a s} \subseteq X^{s s, f g} \subseteq X^{\overline{s s}}=X^{n s s} .
$$

The stable sets $X^{\bar{s}}, X^{\text {lts }}=X^{m s}=X^{m s s}$ and $X^{n s}$ admit quasi-projective geometric quotients, given by restrictions of the quotient map $q=\pi \circ j \circ i$. The quotient map $q=\pi \circ j \circ i$ restricted to the open subvariety $X^{s s, f g}$ is an enveloped quotient with $q: X^{s s, f g} \rightarrow X / / H$ an enveloping quotient in the sense of Definition 4.2.7. The enveloping quotient $X / / H$ is an open subvariety of $\overline{G \times{ }_{H} X} / / G$ and there is an ample line bundle $L_{H}$ on $X / / H$ which pulls back to a tensor power $L^{\otimes k}$ of the line bundle $L$ for some $k>0$, such that for any $m>0$ the sections of $L_{H}^{\otimes m}$ on $X / / H$ are precisely the $H$-invariant sections of the tensor power $L^{\otimes k m}$ of $L$ on $X^{s s, f g}$.

Remark 5.3.2. The main content of this theorem can be summarized in the following diagram

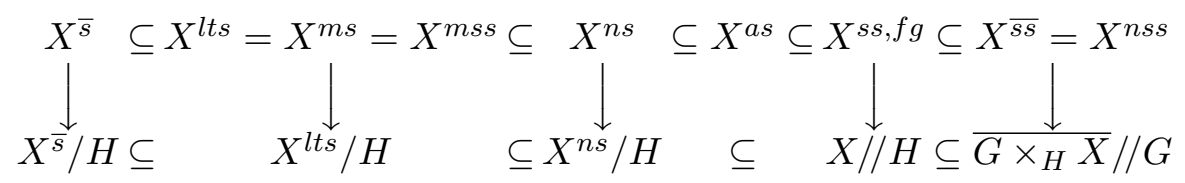

in which all the inclusions are open and all the vertical morphisms are restrictions of $\pi:\left(\overline{G \times{ }_{H} X}\right)^{s s} \rightarrow \overline{G \times_{H} X} / / G$, while every vertical morphism except the last is also a restriction of the map of schemes $\left.q: X^{n s s} \rightarrow \operatorname{Proj}\left(k[X]^{H}\right)\right)$ induced by the inclusion of $k[X]^{H}$ in $k[X]$. Note that everything here is a quasi-projective variety with the exception of $\operatorname{Proj}\left(k[X]^{H}\right)$, which is not a variety unless $k[X]^{H}$ is finitely generated as a $k$-algebra. 
Proof. By definition $X^{s s, f g} \subseteq X^{n s s}$ and $X^{n s} \subseteq X^{a s}$. Lemma 4.2.11 shows that $X^{a s} \subseteq X^{s s, f g}$ and $X^{l t s} \subseteq X^{n s}$ by Definition 4.2.3. Lemma 5.1.7 gives $X^{m s}=$ $X^{m s s}$ and by Proposition 5.1.10, $X^{\text {lts }}=X^{m s}$.

Let $x \in X^{\bar{s}}$ and embed $X$ in $\overline{G \times{ }_{H} X}$ in the natural way. Then for some $G$-invariant $F$ restricting to an $H$-invariant $f$ on $X$ there is an affine subvariety $\left(\overline{G \times{ }_{H} X}\right)_{F}$ containing $x$ on which the $G$ action is proper, and which admits a geometric quotient $\pi$ to $\left(\overline{G \times{ }_{H} X}\right)_{F} / / G$. The boundary in $\left(\overline{G \times{ }_{H} X}\right)_{F}$ is a $G-$ invariant closed set, so the restriction of $\pi$ to the complement of the boundary (that is, to $\left.\left(G \times_{H} X\right)_{F}=G \times_{H} X_{f}\right)$ is also a geometric quotient; furthermore the restricted action is proper because properness is a local property on the base, and hence, since it is set-theoretically free, it is in fact scheme-theoretically free $[13, \S 6.3$ Lemma 8]. It follows from [48, Proposition 0.9] that $\pi$ restricted to $G \times_{H} X_{f}$ is a principal $G$-bundle, and hence by étale descent the morphism $q: X_{f} \rightarrow\left(G \times_{H} X_{f}\right) / G=X_{f} / H$ is a principal $H$-bundle. In particular, $x \in X^{l t s}$.

It is clear that $X^{\overline{s s}} \subseteq X^{n s s}$ because any $G$-invariant section $F$ over $\overline{G \times{ }_{H} X}$ restricts to a $G$-invariant section over $G \times_{H} X$ and hence to an $H$-invariant section $f$ over $X$. Now we argue the reverse containment. By Definition 5.2.5, there exists a finite fully separating set of $H$-invariants $\left\{f_{i}: 1 \leq i \leq r\right\}$ defining $G$-invariant sections $F_{i}$ which extend over the reductive envelope $\overline{G \times{ }_{H} X}$ such that $\left(\overline{G \times_{H} X}\right)_{F_{i}}$ is affine. Thus $\cup_{i} X_{f_{i}} \subseteq X^{\overline{s s}}$. By Definition 5.2.2, given $x \in X$, all the invariant sections $f \in k[X]^{H}$ vanish at $x$ if and only if $f_{i}(x)=0$ for all $i$. Thus $X^{n s s}=\bigcup_{i=1}^{r} X_{f_{i}} \subseteq X^{\overline{s s}}$.

$X^{n s}$ has a geometric quotient by Proposition 4.2.2, and hence the same is true of any $G$-invariant open subset of $X^{n s}$, while $q: X^{s s, f g} \rightarrow X / / H$ is an enveloping quotient by Proposition 4.2.9. It follows from the proof of Proposition 4.2.9 together with condition (ii) in the definition 5.2.2 of a finite fully separating set of invariants that for some $m, M>0$ there exist $H$-invariants $f_{0}, \ldots, f_{M} \in$ $H^{0}\left(X, L^{\otimes m}\right)^{H}$ such that

$$
x \mapsto\left[f_{0}(x): \ldots: f_{M}(x)\right]
$$

induces an embedding of $X / / H$ as a quasi-projective subvariety of $\mathbb{P}^{M}$, and moreover $f_{0}, \ldots, f_{M}$ extend to $G$-invariant sections $F_{0}, \ldots, F_{M}$ of $\left(L^{\prime}\right)^{\otimes m}$ over $\overline{G \times{ }_{H} X}$. Since $k\left[\overline{G \times{ }_{H} X}\right]^{G}$ is finitely generated, by adding restrictions of $G$ invariants over $\overline{G \times{ }_{H} X}$ to $f_{0}, \ldots, f_{M}$ we can assume that $F_{0}, \ldots, F_{M}$ define an embedding

$$
y \mapsto\left[F_{0}(y): \ldots: F_{M}(y)\right]
$$

of $\left(\overline{G \times{ }_{H} X}\right) / / G=\operatorname{Proj}\left(k\left[\overline{G \times{ }_{H} X}\right]^{G}\right)$ as a projective subvariety of the same projective space $\mathbb{P}^{M}$. The latter embedding is the composition of the first embedding with the natural map $X / / H \rightarrow\left(\overline{G \times_{H} X}\right) / / G$, so the natural map embeds $X / / H$ as a subvariety of $\left(\overline{G \times \times_{H} X}\right) / / G$. 
Finally, the existence of an ample line bundle $L_{H}$ on $X / / H$ with the desired properties is proved in Proposition 4.2.9. The identification between invariant sections of $L$ over $X^{s s, f g}$ and sections of $L_{H}$ over $X / / H$ follows trivially by restriction and patching.

Remark 5.3.3. Recall that a fine reductive envelope always exists for any linear $H$-action on a projective variety $X$ by Proposition 5.2.8 and Remark 5.2.6. A choice of fine reductive envelope $\overline{G \times{ }_{H} X}$ provides a projective completion

$$
\overline{X / / H}=\overline{G \times{ }_{H} X} / / G
$$

of the enveloping quotient $X / / H$, which in general depends on the choice of reductive envelope. Note however that when $k[X]^{H}$ is finitely generated then $X / / H=\operatorname{Proj}\left(k[X]^{H}\right)$ is itself projective by Proposition 4.2.10 and hence

$$
X / / H=\overline{G \times H X} / / G
$$

for any fine reductive envelope $\overline{G \times_{H} X}$.

Remark 5.3.4. The Hilbert-Mumford criterion from reductive GIT (see Remark 3.2.5) can be used (at least when $L^{\prime}$ is ample, but see also [28]) to determine $X^{\bar{s}}$ and $X^{\overline{s s}}$ and to analyze the orbit structure of $X^{\overline{s s}} \backslash X^{\bar{s}}$ and the quotient map $q: X^{\overline{s s}} \rightarrow X / / H$ (cf. [39]), which leads in examples to identification of the intermediate stable and semistable sets $X^{m s}=X^{m s s}=X^{l t s} \subseteq X^{n s} \subseteq X^{a s} \subseteq$ $X^{s s, f g}$. In especially good situations we have $X^{\overline{s s}}=X^{\bar{s}}$ (as in the example in the final section $\S 6$ below with $n$ odd) and then

$$
X^{\bar{s}}=X^{m s}=X^{m s s}=X^{l t s}=X^{n s}=X^{a s}=X^{s s, f g}=X^{\overline{s s}} .
$$

Theorem 5.3.5. If $\overline{G \times{ }_{H} X}$ is normal and together with a line bundle $L^{\prime}$ provides a fine strong reductive envelope for the linear $H$-action on $X$, then $X^{\bar{s}}=X^{m s}$ and $X^{s s, f g}=X^{n s s}$.

Proof. We show that $X^{m s} \subseteq X^{\bar{s}}$. Consider $x \in X^{m s}=X^{m s s}$ and embed $X$ in $G \times_{H} X$ in the natural way. Then there exists an $F \in H^{0}\left(G \times_{H} X, L^{\otimes m}\right)^{G}$ for some $m>0$ such that $F(x) \neq 0$ and $\left(G \times_{H} X\right)_{F}$ is affine and the action of $G$ on $\left(G \times_{H} X\right)_{F}$ is closed with all stabilizers of dimension 0 . The section $F$ extends to a $G$-invariant section over the reductive envelope of the $H$-action on $X$ and $F$ vanishes on every codimension 1 component $D_{j}$ of the boundary. We claim that $\left(\overline{G \times{ }_{H} X}\right)_{F}=\left(G \times_{H} X\right)_{F}$ : they are both affine, so they are determined by their coordinate rings, and they differ at most in codimension 2 , so by normality their coordinate rings agree; therefore since one is contained in the other, they must in fact be equal. Thus $x \in X^{\bar{s}}$.

We prove now that $X^{n s s} \subseteq X^{s s, f g}$. Consider a finite fully separating set $S=$ $\left\{f_{i}: 1 \leq i \leq r\right\}$ associated to the reductive envelope, and label the corresponding $G$-invariant sections of powers of $L^{\prime}$ over $\overline{G \times{ }_{H} X}$ by $F_{i}$. Then $x \in\left(\overline{G \times{ }_{H} X}\right)_{F_{i}}$ for 
one of these $G$-invariants $F_{i}$. Since $G$ is reductive and $\left(\overline{G \times_{H} X}\right)_{F_{i}}$ is an affine variety, it has a finitely generated ring of invariants. Furthermore, by the definition of a strong reductive envelope, each $F_{i}$ vanishes on the codimension 1 components $D_{j}$ of the boundary of $G \times_{H} X$ in $\overline{G \times{ }_{H} X}$. It follows that the complement of the quasi-affine $\left(G \times_{H} X\right)_{F_{i}}$ in the affine variety $\overline{G \times}_{H} X_{F_{i}}$ has codimension 2 . Thus by normality we have $k\left[X_{f}\right]^{H}=k\left[\left(G \times_{H} X\right)_{F}\right]=k\left[\left(\overline{G \times{ }_{H} X}\right)_{F}\right]^{G}$ which is finitely generated, so $x \in X^{s s, f g}$. The result now follows from Theorem 5.3.1.

We will see in $\S 5.3 .1$ that fairly mild conditions on the singularities of a completion $\overline{G \times{ }_{H} X}$ are sufficient to prove the existence of a fine strong reductive envelope.

Remark 5.3.6. Let $X$ be an affine variety; then we can define $q: X^{s s, f g} \rightarrow$ $X / / H \subseteq \operatorname{Spec}\left(k[X]^{H}\right)$ by analogy with the definition of $q: X^{s s, f g} \rightarrow X / / H \subseteq$ $\operatorname{Proj}\left(k[X]^{H}\right)$ when $X$ is projective, and $X / / H$ is a quasi-affine variety with coordinate ring $k[X / / H] \cong k\left[X^{s s, f g}\right]^{H}$. When we have an ample strong reductive envelope which is normal then by Theorem 5.3.5 $X^{s s, f g}=X^{n s s}$ where $X^{n s s}=X$ since $X$ is affine (cf. Remark 3.2.12), so $X / / H$ is an explicit construction of Winkelmann's quasi-affine "quotient" variety [60], and the quotient morphism $q: X \longrightarrow X / / H$ plays the rôle of the morphism (rather than just a rational map) Winkelmann hoped for.

Definition 5.3.7. Let $X$ be a projective variety equipped with a linear $H$-action. We say that a point $x \in X$ is stable if $x \in X^{m s}$ and that it is semistable if $x \in X^{s s, f g}$.

5.3.1. Constructing strong reductive envelopes. Although Proposition 5.2.8 guarantees the existence of an ample (and hence fine) reductive envelope, its method of construction requires us to identify a finite fully separating set of invariants, and it is not very clear how to do this in practice. The difficulty in building a fine reductive envelope lies in the trade-offs between the choice of completion, finding a line bundle so that enough sections extend, and guaranteeing that the $F=0$ complements are affine. In this subsection we discuss how, for a fixed sufficiently nice (for example, smooth) completion, one can find a line bundle so that the sections extend; when this line bundle is ample, we get a strong ample reductive envelope. The technique, with slight adjustment, will be applied in $\S 5.3 .2$ and $\S 5.3 .3$.

Definition 5.3.8. Let $Y$ be a normal quasi-projective variety. We say that a completion $\bar{Y}$ is gentle if it is normal and some integral multiple of each boundary Weil divisor is Cartier.

Remark 5.3.9. Over a characteristic 0 field, as we have assumed from the start, a gentle completion can always be arranged by equivariant resolution of singularities. 
Given a gentle $G$-equivariant completion $\overline{G \times{ }_{H} X}$ of $G \times_{H} X$, some positive tensor power of any line bundle on $G \times_{H} X$ will extend non-canonically across the boundary. One can check the appropriate cocycle condition [48, proof of Converse 1.13, page 41] to verify that in fact the $G$-linearization extends as well. If the boundary is codimension at least 2 , then by normality all invariant sections extend uniquely to invariant sections over the whole of $\overline{G \times{ }_{H} X}$. If the boundary has codimension 1 components, the basic idea is to weight them heavily enough that any given invariant section extends uniquely over the boundary, vanishing on the codimension 1 components.

To make this precise, let $\left\{D_{j}: 1 \leq j \leq r\right\}$ denote the collection of codimension 1 components of the boundary of $G \times_{H} X$ in $\overline{G \times{ }_{H} X}$, let $L$ be the $G$-linearization on $G \times_{H} X$ discussed in $S 5.1$, and let $L^{\prime}$ be some chosen extension to a $G$ linearization over a projective completion $\overline{G \times{ }_{H} X}$. Denote by $L_{N}^{\prime}$ the induced $G$ linearization on $L^{\prime}\left[N \sum_{j=1}^{r} D_{j}\right]$ when $N$ is such that $N D_{j}$ is Cartier for $1 \leq j \leq r$. Then we have the following proposition.

Proposition 5.3.10. Let $\overline{G \times{ }_{H} X}$ be a gentle $G$-equivariant completion of $G \times_{H}$ $X$, with a $G$-linearization $L^{\prime}$ of the $G$-action which extends the given linearization $L$. Given a finite fully separating set $S$ of invariants on $X$, then $\left(\overline{G \times{ }_{H} X}, L_{N}^{\prime}\right)$ is a strong reductive envelope with respect to $S$ for suitable sufficiently large $N$. If moreover $\left(\overline{G \times{ }_{H} X}, L^{\prime}\right)$ is a fine reductive envelope with respect to $S$ then $\left(\overline{G \times{ }_{H} X}, L_{N}^{\prime}\right)$ is a fine strong reductive envelope with respect to $S$, and hence Theorems 5.3.1 and 5.3.5 apply.

Proof. (Cf. [48, proof of Converse 1.13, page 41]). For any given $f \in S$, there exists an $N_{f}$ such that $f$ extends to a section of $L_{N_{f}}^{\prime}$ over the codimension 1 components $D_{j}$ of the boundary of $G \times_{H} X$ in $\overline{G \times_{H} X}$, and thus by normality extends to an invariant section $F$ over the whole of $\overline{G \times{ }_{H} X}$. We can identify $H^{0}\left(\overline{G \times{ }_{H} X}, L_{n}^{\prime}\right)$ with a subspace of $H^{0}\left(\overline{G \times_{H} X}, L_{n+1}^{\prime}\right)$ for any $n$, so that if $f$ extends to a section $F$ of $L_{n}^{\prime}$ then it vanishes on each $D_{j}$ as a section of $L_{n+1}^{\prime}$. Thus taking $N>N_{f}$ forces $F$ to vanish on each of the codimension 1 components $D_{j}$ of the boundary. Consequently, since $S$ is finite, simply take $N>\max _{f \in S}\left(N_{f}\right)$; then $\left(\overline{G \times{ }_{H} X}, L_{N}^{\prime}\right)$ is a strong reductive envelope for the linear $H$-action on $X$.

For the final part, observe that the complement of a Cartier divisor in an affine variety is affine, because any line bundle on an affine variety is ample [27, Example II.7.4.2]. Thus the complement of the $D_{j}$ in each affine $\left(\overline{G \times{ }_{H} X}\right)_{F}$ is affine, and the result follows.

Remark 5.3.11. Unfortunately the following three properties do not in general hold simultaneously, although we can arrange any two of them: the completion $\overline{G \times{ }_{H} X}$ being gentle, the line bundle $L^{\prime}$ being ample, and the completion 
together with the line bundle forming a reductive envelope (that is, enough invariants extending). More concretely, if $X$ is affine and there exists a gentle ample reductive envelope, then $k[X]^{H}$ must be finitely generated, since by Remark 5.2.10, it suffices to show there exists a fine strong reductive envelope with respect to a finite fully separating set $S$ of invariants which includes a nonzero constant function over $X$. Because the completion is gentle, we can construct $L_{N}^{\prime}$ as in Proposition 5.3.10 above for any such $S$, and for large enough $N$ it defines a fine strong reductive envelope.

In particular, the Nagata counter-examples admit no gentle ample reductive envelopes.

Observe that essentially the same argument shows that if $G / H$ has a gentle $G$-equivariant affine completion $\overline{G / H}^{\text {aff }}$ then $G / H$ can be represented as a codimension 2 complement in an affine $G$-variety, and hence $k[G / H]=k[G]^{H}$ is finitely generated (cf. [25, §4]).

Remark 5.3.12. Suppose that $L^{\prime}$ is chosen to be ample in Proposition 5.3.10. If the line bundles $L_{N}^{\prime}$ are also ample for large $N$ (for example, if the divisors given by positive integral multiples of the $D_{j}$ are ample), then we can compare the $G$-linearizations $\left(\overline{G \times{ }_{H} X}, L_{N}^{\prime}\right)$ for different $N \geq 0$ using the theory of variation of GIT quotients $[56,12]$. Since GIT is unaffected when we replace a line bundle by any positive tensor power of itself we can consider $L_{N}^{\prime}$ for all positive rational values of $N$. We know $[56,12]$ that the interval $(0, \infty)$ can be divided into (rational) subintervals $I_{j}$ such that when $N$ lies in the interior of a subinterval $I_{j}$ the GIT quotient (and the stable and semistable sets) defined with respect to the linearization $L_{N}^{\prime}$ depends only on the subinterval in which $N$ lies. Moreover if $N$ lies on the boundary between two subintervals then there is a nonempty set of points which are semistable with respect to the linearization $L_{N}^{\prime}$ (for this particular $N$ ) but not semistable for the whole family, having as stabilizer a reductive subgroup $R$ of dimension at least one in $G$. Any maximal torus of $R$ acts with zero weights on the fibres of $L_{N}^{\prime}$ at all semistable fixed points of $R$. Up to conjugacy only finitely many subgroups can occur as stabilizers, and their fixed point sets have only finitely many connected components. From this and the requirement of zero weights on the fibres of $L_{N}^{\prime}$, it follows that only finitely many positive $N$ can occur as the boundary between two subintervals $I_{j}$, and hence that there are only finitely many such subintervals.

This proves:

Lemma 5.3.13. For $N \geq 0$ let $\left(\overline{G \times_{H} X}, L_{N}^{\prime}\right)$ be an ample strong reductive envelope for a linear $H$-action on a projective variety $X$. Then the GIT quotient $\overline{G \times{ }_{H} X} / / G$ and the stable and semistable sets in $\overline{G \times{ }_{H} X}$ defined with respect to the $G$-linearizations $L_{N}^{\prime}$ are independent of $N$ when $N$ is sufficiently large. 
5.3.2. Important case: $H$-action extends to $G$-action. If a linear $H$-action on a projective variety $X$ extends to a linear reductive $G$-action on $X$, then since $G \times{ }_{H} X \cong(G / H) \times X$ (cf. Remark 5.1.5), the problem of constructing an ample reductive envelope reduces to understanding completions of $G / H$ and extensions of the trivial line bundle over these completions. Since $G / H$ is quasi-affine, the natural choice is to take a $G$-equivariant affine closure $\overline{G / H}$ aff , and then projectively complete with the hypersurface at infinity. Many practical applications of non-reductive GIT, for example to moduli spaces of hypersurfaces in toric varieties, fall into this setting. Two scenarios work out especially well:

- if a $G$-equivariant affine closure $\overline{G / H}^{\text {aff }}$ contains $G / H$ as a codimension 2 complement, and

- if a $G$-equivariant affine closure $\overline{G / H}^{\text {aff }}$ is nonsingular.

These are both special cases of a third:

- if a $G$-equivariant affine closure $\overline{G / H}^{\text {aff }}$ is gentle (that is, given a Weil divisor in the boundary, some integer multiple is Cartier),

though in fact this apparent extra generality is spurious, as we observed in Remark 5.3.11: the existence of a gentle $G$-equivariant affine closure implies that $G / H$ can be embedded as a codimension 2 complement in an affine $G$-variety, which in turn implies that $k[G / H]=k[G]^{H}$ is finitely generated [25, §4], and by the Borel transfer principle that

$$
k[X]^{H} \cong k[(G / H) \times X]^{G}
$$

is finitely generated whenever the linear action of $H$ on $X$ extends to a linear $G$-action.

Note that the affine closure $\overline{G / H}^{\text {aff }}$ of $G / H$ can always be chosen to be normal, so we assume that throughout. We will see that any of the three conditions above implies the existence of a strong ample reductive envelope when the $H$-action extends to a linear $G$-action, and hence, by Theorem 5.3.5, that $X^{\bar{s}}=X^{m s}$ and $X^{s s, f g}=X^{\overline{s s}}=X^{n s s}$ for such an envelope, while $X / / H=\operatorname{Proj}\left(k[X]^{H}\right)$ by Proposition 4.2.10.

Let $\overline{G / H}$ denote the normalized projective completion $\operatorname{Proj}\left(k\left[\overline{G / H}^{\text {aff }}\right] \otimes k[x]\right)$. Let $L^{\prime}$ denote the $G$-linearization obtained by taking the hyperplane line bundle on $\overline{G / H}$ (with its natural $G$-linearization) tensored with the given $G$-linearized ample line bundle $L$ on $X$. Let $D_{\infty}$ represent the hypersurface at infinity in $\overline{G / H}$. Note that $D_{\infty}$ is a Cartier divisor on $\overline{G / H}$ (corresponding to the hyperplane line bundle), so $\overline{G / H}$ is a gentle completion of $G / H$ if and only if $\overline{G / H}$ aff is gentle. 
Lemma 5.3.14. If the given linear $H$-action on $X$ extends to a linear $G$-action, and if $G / H$ has a gentle $G$-equivariant affine closure $\overline{G / H}{ }^{a f f}$, then $\left(\overline{G / H} \times X, L_{N}^{\prime}\right)$ is a strong ample reductive envelope for suitable large $N$, where $L_{N}^{\prime}$ is defined as in Proposition 5.3.10.

Proof. For $L_{N}^{\prime}$ as in Proposition 5.3.10 to be defined requires that $N D_{j}$ should be a Cartier divisor for each codimension 1 component $D_{j}$ of the boundary of $G \times{ }_{H} X$ in $\overline{G \times{ }_{H} X}$. Since any line bundle on an affine variety is ample, and since the hypersurface at infinity is an ample divisor, $L_{N}^{\prime}$ is ample for every such $N$. The result now follows immediately from Proposition 5.3.10.

Proposition 5.3.15. If the linear $H$-action on a projective variety $X$ extends to a linear $G$-action, and if $\overline{G / H}$ aff is gentle, then $X^{m s}=X^{\bar{s}}$ and $X^{n s s}=X^{\overline{s s}}=$ $X^{s s, f g}$ has a canonical enveloping quotient $X / / H=\operatorname{Proj}\left(k[X]^{H}\right)$ which is realized as a reductive GIT quotient.

Proof. This follows from Lemma 5.3.14, Proposition 4.2.10 and Theorems 5.3.1 and 5.3.5.

Remark 5.3.16. An even more special case is when $H$ is a maximal unipotent subgroup of $G$; this has been studied by many authors including $[42,43]$ and recently $[26,30]$.

Even when the linear $H$-action fails to extend to a linear $G$-action on $X$, this is true for the ambient projective space $\mathbb{P}^{n}$, so we obtain the following corollary.

Corollary 5.3.17. If $\overline{G / H}^{\text {aff }}$ is gentle, then

$$
X^{\bar{s}}=X \cap\left(\mathbb{P}^{n}\right)^{m s}
$$

is independent of the choice of $\overline{G / H}^{\text {aff }}$.

Proof. Since $(G / H) \times X$ is closed in $(G / H) \times \mathbb{P}^{n}$, by reductive GIT [48, Theorem 1.19] we have $X^{\bar{s}}=X \cap\left(\mathbb{P}^{n}\right)^{\bar{s}}$, and by Proposition 5.3.15 applied to $\mathbb{P}^{n}$ we have $\left(\mathbb{P}^{n}\right)^{\bar{s}}=\left(\mathbb{P}^{n}\right)^{m s}$.

5.3.3. Consequences for finite generation. Let us now assume that $X$ is nonsingular, so that by resolution of singularities we can find a nonsingular (and hence gentle) projective completion $\overline{G \times_{H} X}$. The construction of $L_{N}^{\prime}$ in Proposition 5.3.10 gives us a necessary and sufficient condition for the ring of invariants $k[X]^{H}$ to be finitely generated; when $L_{N}^{\prime}$ is ample, this condition is that for large enough $N$ the codimension 1 components of the boundary of $G \times_{H} X$ in $\overline{G \times{ }_{H} X}$ are all unstable. 
Theorem 5.3.18. Let $X$ be a nonsingular projective variety acted on by a connected unipotent group $H$, and let $L$ be a linearization of the $H$-action with respect to a projective embedding $X \subseteq \mathbb{P}^{n}$ of $X$. Let $H \hookrightarrow G$ be a closed immersion into a reductive subgroup of $S L(n+1 ; k)$. Let $L$ be the induced $G$-linearization over $G \times_{H} X$ and let $L^{\prime}$ be an extension of $L$ over a gentle completion $\left(\overline{G \times{ }_{H} X}\right)$.

Then the ring of invariants $k[X]^{H}$ is finitely generated if and only if there exists $N$ such that, for all $N^{\prime}>N$ for which $L_{N^{\prime}}^{\prime}$ is defined, any $G$-invariant section of a positive tensor power of $L_{N^{\prime}}^{\prime}$ vanishes on every codimension 1 component $D_{j}$ in the boundary of $G \times_{H} X$ in $\overline{G \times{ }_{H} X}$, where $L_{N}^{\prime}=L^{\prime}\left[N \sum_{j} D_{j}\right]$.

Proof. By restriction $k\left[\overline{G \times{ }_{H} X}\right]^{G} \subseteq k\left[G \times_{H} X\right]^{G}=k[X]^{H}$. The forward direction is then a consequence of the construction of Proposition 5.3.10 as follows. For large enough $N$, any given invariant section over $G \times_{H} X$ extends and vanishes on each $D_{j}$. So for large enough $N$ the finitely many generators of the ring of invariants will all vanish on every $D_{j}$, hence every element of $k\left[\overline{G \times{ }_{H} X}\right]^{G}$ vanishes on every $D_{j}$.

The reverse direction follows by proving that for any such $N$ the ring of invariants $k[X]^{H} \cong k\left[G \times_{H} X\right]^{G}$ is isomorphic to the ring of invariants $k\left[\overline{G \times{ }_{H} X}\right]^{G}$, defined with respect to the linearization $L_{N}^{\prime}$, which is finitely generated since $\overline{G \times H} X$ is a projective variety acted on linearly by the reductive group $G$. This isomorphism arises since any invariant section $s$ over $G \times_{H} X$ of $L_{N}^{\prime}$ extends as in the proof of Proposition 5.3.10 above to an invariant section of $L_{N^{\prime}}^{\prime}$ over $\overline{G \times_{H} X}$ for some $N^{\prime}>N$. By hypothesis this section vanishes on each $D_{j}$ and hence defines a section of $L_{N^{\prime}-1}^{\prime}$ extending $s$. Repeating this argument enough times we find that $s$ extends to a section of $L_{N}^{\prime}$. The same argument applies to any invariant section $s$ over $G \times_{H} X$ of a positive tensor power $\left(L_{N}^{\prime}\right)^{\otimes m}$ of $L_{N}^{\prime}$, so we have $k\left[G \times{ }_{H} X\right]^{G} \cong k\left[\overline{G \times{ }_{H} X}\right]^{G}$ as required.

Corollary 5.3.19. In the setting of Theorem 5.3.18, if the $L_{N}^{\prime}$ are ample for all $N^{\prime}>N$, then $k[X]^{H}$ is finitely generated if and only if every $D_{i}$ is unstable for all such $N^{\prime}$.

Proof. For an ample bundle, the complement of the zero set of a section is affine. In particular, given an ample $G$-linearization, the set on which all invariant sections vanish is precisely the unstable set.

Remark 5.3.20. For $H$-actions extending to $G$-actions on a nonsingular projective variety $X$, this necessary and sufficient condition can be made effective by an explicit construction of a suitable projective completion for $G / H$ as in $\S 5.3 .2$, together with a careful analysis of Hilbert-Mumford in this setting (cf. Remark 3.2.5), at least when the bundles $L_{N}^{\prime}$ are ample. When the bundles $L_{N}^{\prime}$ are not ample the analysis of stability is less straightforward (cf. [28]). Unfortunately 
ampleness does not always occur, as can be seen when $H=\mathbb{C}^{+}$and $G=S L(2 ; \mathbb{C})$ and $\overline{G / H}$ is the blow-up of $\mathbb{P}^{2}$ at 0 .

\section{EXAMPLE: $n$ UNORDERED POINTS ON $\mathbb{P}^{1}$}

Let $H=\mathbb{C}^{+}$, identified with the group of upper triangular matrices of the form

$$
\left(\begin{array}{ll}
1 & b \\
0 & 1
\end{array}\right)
$$

in $G L(2 ; \mathbb{C})$, act linearly on $X=\mathbb{P}^{n}=\mathbb{P}\left(\operatorname{Sym}^{n}\left(\mathbb{C}^{2}\right)\right)$ via the standard representation of $G L(2 ; \mathbb{C})$ on $\operatorname{Sym}^{n}\left(\mathbb{C}^{2}\right)$.

Let $G=S L(2 ; \mathbb{C})$; then we can identify $G / H$ with $\mathbb{C}^{2} \backslash\{0\}$ via the usual transitive action of $S L(2 ; \mathbb{C})$ on $\mathbb{C}^{2} \backslash\{0\}$ which extends to a linear action on its projective completion $\mathbb{P}^{2}=\overline{G / H}$ with the point $[1: 1: 0]$ representing the identity coset $H$. Since the linear action of $H$ on $X$ extends to $G$ we have $G \times{ }_{H} X \cong G / H \times X$, and we are in the setting of $\S 5.3 .2$. Since $\mathbb{P}^{2}$ is smooth, this is a gentle completion. Let $L$ denote the hyperplane line bundle on $X=\mathbb{P}^{n}$ and let $L_{2}$ denote the hyperplane line bundle on $\overline{G / H}=\mathbb{P}^{2}$. For any positive integers $p$ and $q$ there is then an induced linearization of the action of $G$ on $\overline{G / H} \times X$ with respect to the line bundle $L^{\otimes p} \otimes L_{2}^{\otimes q}$. Note this is a line bundle of the type denoted by $L_{q}^{\prime}$ in $\S 5.3 .2$. In particular, by Lemma 5.3.14 this provides a strong ample reductive envelope for large enough $q$. By Theorem 5.3.5 this means $X^{\bar{s}}=X^{m s}$ and $X^{n s s}=X^{s s, f g} \stackrel{\text { def }}{=} X^{s s}=X^{\overline{s s}}$.

We know that $\mathbb{C}[X]^{H}$ is finitely generated because $G / H$ is a codimension 2 complement in its affine closure $\mathbb{A}^{2}[25, \S 4]$. We can also see this by applying the finitely generated criterion, Theorem 5.3.18: here there is only one boundary divisor, namely the product of the line at infinity with $X$; by the Hilbert-Mumford numerical criterion (see Remark 3.2.3) it is easy to see that this is unstable, and in particular that all the invariants vanish there, for sufficiently large $q$.

By the Hilbert-Mumford numerical criterion, a point of $\mathbb{P}\left(\operatorname{Sym}^{n}\left(\mathbb{C}^{2}\right)\right) \times\{[1$ : $1: 0]\} \subseteq \mathbb{P}\left(\operatorname{Sym}^{n}\left(\mathbb{C}^{2}\right)\right) \times \mathbb{P}^{2}$, represented by an unordered sequence $p_{1}, \ldots, p_{n}$ of points in $\mathbb{P}^{1}$, is stable for this linear action of $G$ provided that

- strictly fewer than $\frac{n}{2}+\frac{q}{p}$ of the points $p_{1}, \ldots, p_{n}$ coincide anywhere on $\mathbb{P}^{1}$, and

- strictly fewer than $\frac{n}{2}$ of the points $p_{1}, \ldots, p_{n}$ coincide at $[1: 0]$;

it is $G$-semistable unless

- strictly more than $\frac{n}{2}+\frac{q}{p}$ of the points $p_{1}, \ldots, p_{n}$ coincide anywhere on $\mathbb{P}^{1}$, or

- strictly more than $\frac{n}{2}$ of the points $p_{1}, \ldots, p_{n}$ coincide at $[1: 0]$. 
When $q$ is large compared with $p$ then the first condition is vacuous in each case, and so a point of $\mathbb{P}\left(S y m^{n}\left(\mathbb{C}^{2}\right)\right)$ represented by an unordered sequence $p_{1}, \ldots, p_{n}$ of points in $\mathbb{P}^{1}$ is in $X^{\bar{s}}=X^{s}$ provided that strictly fewer than $n / 2$ of the points coincide at $[1: 0]$, and is in $X^{\overline{s s}}=X^{s s}$ unless strictly more than $n / 2$ coincide there.

Thus when $n$ is odd semistability and stability coincide and we have a geometric quotient $X^{s} / H=X^{s s} / H$ which is an open subset of $X / / H=\operatorname{Proj}\left(k[X]^{H}\right) \cong$ $(\overline{G / H} \times X) / / G$; its complement can be identified with the reductive quotient $X / / G$. Observe that a point of $X=\mathbb{P}\left(\operatorname{Sym}^{n}\left(\mathbb{C}^{2}\right)\right)$ represented by an unordered sequence $p_{1}, \ldots, p_{n}$ of points in $\mathbb{P}^{1}$ is $G$-stable provided that strictly fewer than $n / 2$ of the points coincide anywhere on $\mathbb{P}^{1}$, and is $G$-semistable unless strictly more than $n / 2$ coincide anywhere on $\mathbb{P}^{1}$.

When $n$ is even we have a geometric quotient $X^{s} / H$ which is an open subvariety of $X / / H \cong(\overline{G / H} \times X) / / G$, again with complement $X / / G$, but now the image of $X^{s s}$ in $X / / H$ is not a subvariety: it is the union of the open subvariety $X^{s} / H$ and the closed subvariety - which is in fact a point - $(X / / G) \backslash\left(X^{s, G} / G\right)$ where $X^{s, G}$ is the stable set for the action of $G$ on $X$.

When $n$ is odd $X^{s}=X^{n s}=X^{a s}=X^{s s}$. Indeed, whenever $n>2$ then $X^{s}=X^{n s}=X^{a s}$ : by continuity, given the $\mathbb{C}^{+}$-action on $\mathbb{P}^{1}$ no $\mathbb{C}^{+}$-invariant can distinguish among orbits consisting of points which correspond to configurations with $n / 2$ points at [1:0], and if $n>2$ this set is more than 1-dimensional (the dimension of $\mathbb{C}^{+}$).

Note that the algorithm based on flattening stratifications described in [21] produces a set of stable points much smaller than $X^{s}$; for these $\mathbb{C}^{+}$-actions the algorithm removes the hyperplane $x_{0}=0$, where $x_{0}$ is the unique $\mathbb{C}^{+}$-invariant coordinate function.

6.1. $n=3$ and 4. Let $x_{0}, \ldots, x_{n}$ be the usual coordinates on $S y m^{n}\left(\mathbb{C}^{2}\right)$, so that if $\left[x_{0}, \ldots, x_{n}\right] \in \mathbb{P}\left(\operatorname{Sym}^{n}\left(\mathbb{C}^{2}\right)\right)$ corresponds to an unordered sequence $p_{1}, \ldots, p_{n}$ of points in $\mathbb{P}^{1}$ then $x_{0}, \ldots, x_{n}$ are the coefficients of a homogeneous polynomial of degree $n$ in two variables whose roots are $p_{1}, \ldots, p_{n}$.

When $n$ is small, using Gröbner basis techniques (in particular, an adaptation of an algorithm in [15, Chapter 1]) we can explicitly find generators for the ring of $H$-invariants in the polynomial ring

$$
\bigoplus_{j \geq 0} H^{0}\left(X, L^{\otimes j}\right)=\mathbb{C}\left[x_{0}: \ldots: x_{n}\right] .
$$

For $n=3$ we have four generators

$$
x_{0}, \quad x_{0} x_{2}-x_{1}^{2}, \quad x_{1}^{3}+\frac{1}{2} x_{0}^{2} x_{3}-\frac{3}{2} x_{0} x_{1} x_{2}
$$


and

$$
2 x_{0} x_{1} x_{2} x_{3}-\frac{1}{3} x_{0}^{2} x_{3}^{2}+x_{1}^{2} x_{2}^{2}-\frac{4}{3} x_{1}^{3} x_{3}-\frac{4}{3} x_{0} x_{2}^{3}
$$

for the invariants, so all the invariants vanish if and only if

$$
x_{0}=x_{1}=0,
$$

or equivalently if and only if at least two of the three points $p_{1}, p_{2}, p_{3}$ coincide at $[1: 0]$, as expected from the analysis above. The enveloping quotient $X / / H=\operatorname{Proj}\left(k[X]^{H}\right)$ is a degree 6 hypersurface in the weighted projective space $\mathbb{P}(1,2,3,4)$ with equation

$$
3 y_{0}^{2} y_{3}=4 y_{1}^{3}-4 y_{2}^{2}
$$

and $X^{s} / H=X^{s s} / H$ is the complement of the point $[0: 0: 0: 1]$ in this hypersurface.

When $n=4$ we have five generators

$$
\begin{gathered}
x_{0}, \quad x_{0} x_{2}-x_{1}^{2}, \quad x_{2}^{2}+\frac{1}{3} x_{0} x_{4}-\frac{4}{3} x_{1} x_{3}, \\
x_{1}^{3}+\frac{1}{2} x_{0}^{2} x_{3}-\frac{3}{2} x_{0} x_{1} x_{2},
\end{gathered}
$$

and

$$
x_{2}^{3}-2 x_{1} x_{2} x_{3}+x_{0} x_{3}^{2}+x_{1}^{2} x_{4}-x_{0} x_{2} x_{4}
$$

so all the invariants vanish if and only if

$$
x_{0}=x_{1}=x_{2}=0,
$$

or equivalently if and only if at least three of the two points $p_{1}, p_{2}, p_{3}, p_{4}$ coincide at [1:0], as expected. In this case the boundary $(X / / H) \backslash\left(X^{s} / H\right)$ of $X^{s} / H$ in $X / / H$ is $\mathbb{P}^{1}$ and the image of $X^{s s}$ in $X / / H$ is the union of the open subset $X^{s} / H$ and the point $\infty$ in its complement $\mathbb{P}^{1}$. The enveloping quotient $X / / H=\operatorname{Proj}\left(k[X]^{H}\right)$ is a degree 6 hypersurface in the weighted projective space $\mathbb{P}(1,2,2,3,3)$ with equation

$$
4 y_{3}^{2}-4 y_{1}^{3}-y_{0}^{3} y_{4}+3 y_{0}^{2} y_{1} y_{2}=0 .
$$

Remark 6.1.1. For $n>4$ the problem of computing invariants rapidly becomes unmanageable. Indeed already for $n=5$ a computation in Singular produces 15 invariant generators and is unable to verify that it is a complete set. The difficulty is not surprising, as this problem is closely related to the well-known one of producing a generating set of $S L_{2}$-invariants for binary forms of degree $n+2$, which is very hard for any $n+2>6$. 
6.2. Cohomology of quotients. We can study the rational intersection cohomology [19, 20] of any reductive GIT quotient $Y / / G$, where $Y$ is a projective variety, by stratifying $Y$ and using equivariant (intersection) cohomology $[31,32,34,35,36,37,38]$. These methods apply in particular to any ample reductive envelope $Y=\overline{G \times{ }_{H} X}$ of a linear $H$-action on a projective variety $X$ over $k=\mathbb{C}$, allowing us to investigate the intersection cohomology of the projective completion $Y / / G=\overline{X / / H}$ of the enveloping quotient $X / / H$; when, as in the examples we are considering, $k[X]^{H}$ is a finitely generated $k$-algebra so that $X / / H=\operatorname{Proj}\left(k[X]^{H}\right)$ is projective, this is the intersection cohomology of the enveloping quotient $X / / H$ itself.

The simplest situation is when $Y=\overline{G \times_{H} X}$ is nonsingular; this holds in our examples since $Y=\mathbb{P}^{2} \times \mathbb{P}^{n}$. Then we have a $G$-equivariantly perfect stratification $\left\{S_{\beta}: \beta \in \mathcal{B}\right\}$ of $Y$ by $G$-invariant locally closed nonsingular subvarieties of $Y$ with $S_{0}=Y^{s s}$ as an open stratum, so that

$$
P_{t}^{G}\left(Y^{s s}\right)=P_{t}^{G}(Y)-\sum_{\beta \neq 0} t^{2 d_{\beta}} P_{t}^{G}\left(S_{\beta}\right)
$$

where $P_{t}^{G}(Z)=\sum_{i \geq 0} t^{i} \operatorname{dim} H_{G}^{i}(Z ; \mathbb{Q})$ is the $G$-equivariant Poincaré series of a $G$ space $Z$ and $d_{\beta}$ is the (complex) codimension of $S_{\beta}$ in $Y$ [35]. When $G=S L(2 ; \mathbb{C})$ and $Y=\mathbb{P}^{2} \times \mathbb{P}^{n}$ then the stratification $\left\{S_{\beta}: \beta \in \mathcal{B}\right\}$ is given by

$$
S_{0}=Y^{s s}
$$

and for $n / 2<j \leq n$

$$
S_{j, 0}=\left\{(w, x) \in \mathbb{P}^{2} \times \mathbb{P}^{n}: x \text { represents } n \text { points of } \mathbb{P}^{1}\right.
$$

exactly $j$ of which coincide at $[a: b]$ where $y=[1: t a: t b]$ for some $t \in \mathbb{C}\}$ with codimension $j$ in $Y$, and for $0 \leq j \leq n$

$$
S_{j, 1}=\left\{(w, x) \in \mathbb{P}^{2} \times \mathbb{P}^{n}: x \text { represents } n \text { points of } \mathbb{P}^{1}\right.
$$

exactly $j$ of which coincide at $[a: b]$ where $y=[0: a: b]\}$

with codimension $j+1$ in $Y$. Equation (2) gives us

$$
\begin{gathered}
P_{t}^{G}\left(Y^{s s}\right)=\left(1+t^{2}+t^{4}\right)\left(1+t^{2}+\cdots+t^{2 n}\right)\left(1-t^{4}\right)^{-1} \\
\quad-\sum_{\frac{n}{2}<j \leq n} t^{2 j}\left(1-t^{2}\right)^{-1}-\sum_{0 \leq j \leq n} t^{2 j+2}\left(1-t^{2}\right)^{-1} .
\end{gathered}
$$

When $n$ is odd we have $Y^{s s}=Y^{s}$ and so $Y / / G=Y^{s s} / G=Y^{s} / G$ and the Poincaré polynomial $P_{t}(X / / H)=\sum_{i \geq 0} t^{i} \operatorname{dim} H^{i}(X / / H ; \mathbb{Q})$ of the enveloping quotient $X / / H=Y / / G$ is given by

$$
\begin{aligned}
P_{t}(X / / H)=P_{t}^{G}\left(Y^{s s}\right)= & {\left[\left(1+t^{2}+t^{4}\right)\left(1+t^{4}+t^{8}+\cdots+t^{2 n-2}\right)-\left(t^{n+1}+t^{n+3}+\cdots+t^{2 n}\right)\right.} \\
& \left.-\left(t^{2}+t^{4}+\cdots+t^{2 n+2}\right)\right]\left(1-t^{2}\right)^{-1} \\
=\left(1+t^{4}+t^{8}\right. & \left.+\cdots+t^{2 n-2}-t^{n+1}-t^{n+3}-\cdots-t^{2 n}\right)\left(1-t^{2}\right)^{-1}
\end{aligned}
$$


$=1+t^{2}+2 t^{4}+2 t^{6}+\cdots+\left[1+\frac{\min (j, n-1-j)}{2}\right] t^{2 j}+\cdots+2 t^{2 n-8}+2 t^{2 n-6}+t^{2 n-4}+t^{2 n-2}$

where [ ] denotes the integer part. Thus $[1+(\min (j, n-1-j)) / 2]$ is the $j$ th Betti number of $X / / H$ when $n$ is odd; this is also the $j$ th intersection Betti number since $X / / H$ is an orbifold and so its rational intersection cohomology is the same as its ordinary rational cohomology. The Poincaré polynomial of the geometric quotient $X^{s} / H=X^{s s} / H=(X / / H) \backslash(X / / G)$ is given by

$$
P_{t}\left(X^{s} / H\right)=P_{t}(X / / H)-t^{4} P_{t}(X / / G)=1+t^{2}+t^{4}+\cdots+t^{n-1} .
$$

We can in fact see this directly, since $X^{s}$ retracts onto $\mathbb{P}^{(n-1) / 2}$ and $H$ is contractible.

When $n$ is even then $Y^{s s} \neq Y^{s}$ and $X / / H=Y / / G$ is not an orbifold, which means that there is more work to do to compute the intersection Betti numbers of $X / / H$. We first find the ordinary Betti numbers of a 'partial desingularization' $\widehat{X / / H}$ of $X / / H$ (see [36]) obtained by blowing up the image in $X / / H=Y / / G$ of the subvariety $Z$ of $Y$ consisting of those $(w, x) \in \mathbb{P}^{2} \times \mathbb{P}^{n}$ with $w=[1: 0: 0]$ (the origin in $\mathbb{C}^{2} \subseteq \mathbb{P}^{2}$ ) and $x$ representing $n$ points on $\mathbb{P}^{1}$, exactly half of which coincide at some $u \in \mathbb{P}^{1}$ and the remaining half coincide at some $v \neq u$. This partial desingularization $\widehat{X / / H}$ (which has only orbifold singularities) is itself a projective completion of the geometric quotient $X^{s} / H$. It can be represented as $\widetilde{X / / H}=\tilde{Y} / / G$ where $\tilde{Y}^{s s}=\tilde{Y}^{s}$ is obtained from $Y^{s s}$ by blowing up along its intersection with $Z$ and removing the proper transform of the subvariety consisting of those $(w, x) \in \mathbb{P}^{2} \times \mathbb{P}^{n}$ with $x$ representing $n$ points on $\mathbb{P}^{1}$ exactly half of which coincide at some $u=[a: b] \in \mathbb{P}^{1}$, and with $w=[1: t a: t b]$ for some $t \in \mathbb{C}$. We obtain

$$
\begin{gathered}
P_{t}(\widetilde{X / / H})=P_{t}^{G}\left(\tilde{Y}^{s s}\right) \\
=P_{t}^{G}\left(Y^{s s}\right)+\left(t^{2}+t^{4}+\cdots+t^{2 n-2}\right)\left(1-t^{4}\right)^{-1}-t^{n}\left(1+t^{2}+\cdots+t^{n}\right)\left(1-t^{2}\right)^{-1} \\
=1+2 t^{2}+3 t^{4}+\cdots+(n / 2) t^{n-2}+(n / 2) t^{n}+\cdots+t^{2 n-2} .
\end{gathered}
$$

Finally from this, using the decomposition theorem of [4], we can obtain the intersection Poincaré polynomial $I P_{t}(X / / H)=\sum_{i \geq 0} t^{i} \operatorname{dim} I H(X / / H ; \mathbb{Q})$ as

$$
\begin{aligned}
& I P_{t}(X / / H)=P_{t}(\widetilde{X / / H})-\left(t^{2}+t^{4}+2 t^{6}+\cdots+\left[\frac{n}{4}\right] t^{n-2}+\left[\frac{n}{4}\right] t^{n}+\cdots+t^{2 n-6}+t^{2 n-4}\right) \\
& =1+t^{2}+2 t^{4}+2 t^{6}+\cdots+[(n+2) / 4] t^{n-2}+[(n+2) / 4] t^{n}+\cdots+2 t^{2 n-6}+t^{2 n-4}+t^{2 n-2} .
\end{aligned}
$$

For more details see [37]. The Poincaré polynomial of the geometric quotient $X^{s} / H$ when $n$ is even is given by

$$
P_{t}\left(X^{s} / H\right)=P_{t}(\widetilde{X / / H})-t^{4} P_{t}(\tilde{X} / / G)=1+t^{2}+t^{4}+\cdots+t^{n-2}
$$

as we can also see directly, since $X^{s}$ retracts onto $\mathbb{P}^{(n-2) / 2}$ and $H$ is contractible. 
We can also compute intersection pairings in $I H(X / / H ; \mathbb{Q})$ and $H^{*}(\widetilde{X / / H} ; \mathbb{Q})$, and the ring structure of $H^{*}(\widetilde{X / / H} ; \mathbb{Q})$ and of $H^{*}(X / / H ; \mathbb{Q})$ when $n$ is odd, using the methods of [31, 32].

Remark 6.2.1. One can work similarly with other (generalized) cohomology theories, like $K$-theory, or motivic cohomology in the sense of Voevodsky [2], at least when $n$ is odd.

\section{REFERENCES}

[1] A. Asok and B. Doran, On unipotent quotients and some smooth $\mathbb{A}^{1}$-contractible schemes, to appear, International Mathematics Research Notices.

[2] A. Asok, B. Doran and F. Kirwan, Equivariant Motivic Cohomology and Quotients, in preparation.

[3] M. Artin, Versal deformations and algebraic stacks, Inv. Math. 27 (1974) 165-189.

[4] A. Beilinson, J. Bernstein, and P. Deligne, Faisceaux pervers. Analysis and topology on singular spaces, I (Luminy, 1981), 5-171, Astrisque, 100, Soc. Math. France, Paris, 1982.

[5] A. Borel, Linear algebraic groups. Second edition. Graduate Texts in Mathematics, 126. Springer-Verlag, New York, 1991.

[6] C.-L. Chai, and A. Neeman, The naturality in Kirwan's decomposition. Manuscripta Math. 97 (1998), no. 4, 429-434.

[7] D. Cox and S. Katz, Mirror symmetry and algebraic geometry. Mathematical Surveys and Monographs, 68. American Mathematical Society, Providence, RI, 1999.

[8] A.-M. Castravet and J. Tevelev, Hilbert's 14-th Problem and Cox Rings, math.AG/0505337.

[9] H. Derksen and G. Kemper, Computational Invariant Theory, Invariant Theory and Algebraic Transformation Groups I, Encyclopaedia of Mathematical Sciences, Volume 130, Springer-Verlag, 2002.

[10] J. Dixmier and M. Raynaud, Sur le quotient d'une variété algébriques par un groupe algébrique, Adv. in Math. Suppl. Stud. 7A (1981) 327-344.

[11] I. Dolgachev, Lectures on invariant theory, London Mathematical Society Lecture Note Series 296, Cambridge University Press, 2003.

[12] I. Dolgachev and Y. Hu, Variation of geometric invariant theory quotients. Inst. Hautes tudes Sci. Publ. Math. No. 87, (1998), 5-56.

[13] D. Edidin and W. Graham, Equivariant intersection theory. Invent. Math. 131 (1998), no. 3, 595-634.

[14] G. Ellingrud and S. Stromme, On the Chow ring of a geometric quotient. Ann. of Math. (2) 130 (1989), no. 1, 159-187.

[15] A. van den Essen, Polynomial automorphisms and the Jacobian conjecture. Progress in Mathematics, 190. Birkhuser Verlag, Basel, 2000.

[16] A. Fauntleroy, Geometric invariant theory for general algebraic groups, Compositio Mathematica 55 (1985) 63-87.

[17] A. Fauntleroy, Categorical quotients of certain algebraic group actions, Illinois J. Math. 27 (1983) 115-124.

[18] A. Fauntleroy and A. Magid, Proper $G_{a}$-actions. Duke Math. J. 43 (1976), no. 4, 723-729.

[19] M. Goresky and R. MacPherson, Intersection homology theory. Topology 19 (1980), no. 2, 135-162.

[20] M. Goresky and R. MacPherson, Intersection homology. II. Invent. Math. 72 (1983), no. 1, 77-129. 
[21] G.-M. Greuel and G. Pfister, Geometric quotients of unipotent group actions, Proc. London Math. Soc. (3) 67 (1993) 75-105.

[22] G.-M. Greuel and G. Pfister, Geometric quotients of unipotent group actions. II. Singularities (Oberwolfach, 1996), 27-36, Progr. Math., 162, Birkhuser, Basel, 1998.

[23] G.-M. Greuel and G. Pfister, Moduli spaces for torsion-free modules on irreducible curve singularities I, J. Algebraic Geometry 2 (1993) 81-135.

[24] G.-M. Greuel, C. Hertling and G. Pfister, Moduli spaces of semiquasihomogeneous singularities with fixed principal part, J. Algebraic Geom. 6 (1997) no. 1, 169-199.

[25] F. Grosshans, Algebraic homogeneous spaces and invariant theory, Lecture Notes in Mathematics, 1673, Springer-Verlag, Berlin, 1997.

[26] V. Guillemin and R. Sjamaar, Convexity theorems for varieties invariant under a Borel subgroup. Pure Appl. Math. Q. 2 (2006), no. 3, 637-653.

[27] R. Hartshorne, Algebraic geometry, Springer, 1977.

[28] J. Hausen, A general Hilbert-Mumford criterion. Ann. Inst. Fourier (Grenoble) 53 (2003), no. $3,701-712$.

[29] K. Hori and J. Walcher, D-branes from matrix factorizations. Strings 04. Part I. C. R. Phys. 5 (2004), no. 9-10, 1061-1070.

[30] Y.Hu, Quotients by Reductive Group, Borel Subgroup, Unipotent Group and Maximal Torus. Pure Appl. Math. Q. 2 (2006), no. 4.

[31] L. Jeffrey and F. Kirwan, Localization for nonabelian group actions. Topology 34 (1995), no. 2, 291-327.

[32] L. Jeffrey, Y.-H. Kiem, F. Kirwan, and J. Woolf, Cohomology pairings on singular quotients in geometric invariant theory. Transform. Groups 8 (2003), no. 3, 217-259.

[33] T. Kambayashi, M. Miyanishi, and M. Takeuchi. Unipotent algebraic groups. SpringerVerlag, Berlin, 1974. Lecture Notes in Mathematics, Vol. 414.

[34] Y.-H. Kiem, Intersection cohomology of quotients of nonsingular varieties. Invent. Math. 155 (2004), no. 1, 163-202.

[35] F. Kirwan, Cohomology of quotients in symplectic and algebraic geometry. Mathematical Notes, 31. Princeton University Press, Princeton, NJ, 1984.

[36] F. Kirwan, Partial desingularisations of quotients of nonsingular varieties and their Betti numbers. Ann. of Math. (2) 122 (1985), no. 1, 41-85.

[37] F. Kirwan, Rational intersection cohomology of quotient varieties. Invent. Math. 86 (1986), no. 3, 471-505.

[38] F. Kirwan, Rational intersection cohomology of quotient varieties. II. Invent. Math. 90 (1987), no. 1, 153-167.

[39] F. Kirwan, Refinements of the Morse stratification of the normsquare of the moment map. The breadth of symplectic and Poisson geometry, 327-362, Progr. Math., 232, Birkhuser Boston, Boston, MA, 2005.

[40] F. Knop, H. Kraft, and T. Vust, The Picard group of a $G$-variety, in Algebraic Transformation Groups and Invariant Theory, DMV Seminar Band 13, Birkh auser, 1989.

[41] J. Kollár, Quotient spaces modulo algebraic groups, Ann. of Math. (2) 145 (1997), no. 1, 33-79.

[42] H. Kraft, Geometrische Methoden in der Invariantentheorie, Viehweg, Braunschweig, 1984.

[43] H. Kraft and C. Procesi, Classical Invariant Theory, A Primer (lecture notes, updated, 2000), unpublished.

[44] L. Makar-Limanov, On the hypersurface $x+x^{2} y+z^{2}+t^{3}=0$ in $C^{4}$ or a $C^{3}$-like threefold which is not $C^{3}$. Israel J. Math. 96 (1996),419-429.

[45] S. Mukai, Counter-example to Hilbert's fourteenth problem for the 3-dimensional additive group, RIMS preprint 1343, Kyoto, 2001. 
[46] S. Mukai, Geometric realization of $T$-shaped root systems and counterexamples to Hilbert's fourteenth problem. Algebraic transformation groups and algebraic varieties, 123-129, Encyclopaedia Math. Sci., 132, Springer, Berlin, 2004.

[47] S. Mukai, Finite generation of the Nagata invariant rings in A-D-E cases, RIMS preprint 1502, Kyoto, May 2005.

[48] D. Mumford, J. Fogarty and F. Kirwan, Geometric invariant theory, 3rd edition, Springer, 1994.

[49] M. Nagata, On the 14-th problem of Hilbert. Amer. J. Math. 81, 1959, 766-772.

[50] P.E. Newstead, Introduction to moduli problems and orbit spaces, Tata Institute Lecture Notes, Springer, 1978.

[51] V. Popov, On Hilbert's theorem on invariants. Dokl. Akad. Nauk SSSR 249 (1979), no. 3, 551-555. English translation: Soviet Math. Dokl. 20 (1979), no. 6, 1318-1322 (1980).

[52] V. Popov and E. Vinberg, Invariant theory, Algebraic geometry IV, Encyclopaedia of Mathematical Sciences v. 55, 1994.

[53] M. Rosenlicht, On quotient varieties and the affine embedding of certain homogeneous spaces. Trans. Amer. Math. Soc. 1011961 211-223.

[54] E. Rousseau, Étude des jets de Demailly-Semple en dimension 3. Ann. Inst. Fourier (Grenoble) 56 (2006), no. 2, 397-421.

[55] C. Seshadri, Quotient spaces modulo reductive algebraic groups, Ann Math 95 (1972) 511556 .

[56] M. Thaddeus, Geometric invariant theory and flips. J. Amer. Math. Soc. 9 (1996), no. 3, 691-723.

[57] R. Thomas, Notes on GIT and symplectic reduction for bundles and varieties, math.AG/0512411.

[58] J. Walcher, Stability of Landau-Ginzburg branes. J. Math. Phys. 46 (2005), no. 8, 082305, $29 \mathrm{pp}$.

[59] J. Winkelmann, On free holomorphic $C$-actions on $C^{n}$ and homogeneous Stein manifolds, Math. Ann. 286 (1990), no. 1-3, 593-612.

[60] J. Winkelmann, Invariant rings and quasiaffine quotients. Math. Z. 244 (2003), no. 1, 163174 .

Brent Doran

School of Mathematics

Institute for Advanced Study

Princeton, NJ 08540, USA

E-mail: doranb@math.ias.edu

Frances Kirwan

Mathematical Institute

University of Oxford

Oxford, OX1 3LB, UK

E-mail: kirwan@maths.ox.ac.uk 